\title{
Screening of Salt Tolerance Capability of Wheat Genotypes under Salt Stress Condition
}

\author{
M. M. Hasan ${ }^{1}$, M. A. Baque ${ }^{1, *}$, M. A. Habib ${ }^{1}$, M. Yeasmin ${ }^{1}$, M. A. Hakim ${ }^{2}$ \\ ${ }^{1}$ Department of Agronomy, Sher-e-Bangla Agricultural University, Bangladesh \\ ${ }^{2}$ Wheat Research Centre, Bangladesh Agricultural Research Institute, Bangladesh
}

Copyright $\mathrm{O} 2017$ by authors, all rights reserved. Authors agree that this article remains permanently open access under the terms of the Creative Commons Attribution License 4.0 International License

\begin{abstract}
To screen salt tolerance wheat genotypes, germination and seedling growth characters were used as screening criteria. 33 wheat genotypes were tested under 5 different salt concentrations $\left(0,5,10,15\right.$ and $\left.20 \mathrm{dSm}^{-1}\right)$ at central laboratory, Sher-e-bangla Agricultural University, Dhaka-1207, during February to March, 2016. The experiment was conducted with a complete randomize design (CRD) with 5 replications. The results of the experiment revealed that, various germination and seedling growth parameters of the wheat genotypes varied significantly under salt stress. A marked reduction of germination rate, shoot and root length, shoot and root dry weight, relative water content, water retention capacity and vigor index was observed with the increasing of salt concentration for most of the wheat genotypes except ESWYT-5, ESWYT-6 and BARI GOM 28. ESWYT-5, ESWYT-6 and BARI GOM 28 showed consistently better performance against salt stress and there were slow linear reduction was observed with the increasing of salt concentration from 0 to $20 \mathrm{dSm}^{-1}$. So considering the above fact, ESWYT-5, ESWYT-6 and BARI GOM 28 could be promising salt stress genotype against moderate saline condition.
\end{abstract}

Keywords Salinity Stress, Wheat Genotypes, Tolerant Genotype, Sensitive Genotypes

\section{Introduction}

By 2050 the world population will be about 9.10 billion, which will be $34 \%$ higher from today and we need to feed another 2.30 billion people with limited resources. Food production must need to be increased about $70 \%$ and to meet this huge demand cereal production will need to increase about 3 billion metric tons from 2.10 billion metric tons today. But in a dilemma, the world agriculture in $21^{\text {st }}$ century faces versatile challenges. Soil salinity is one of the major abiotic stresses which directly affect plant physiology which causes drastic reduction of crop production. World's $25 \%$ cultivable lands are salinity affected among 400 million ha of total land and the salt intrusion scenario is alarmingly increasing. Bangladesh is also not beyond this threat. In Bangladesh the salinity affected area was 83.3 million ha in 1973, 102 million ha in 2000 and in 2009 it has reached up to 105.5 million ha and the area is being expanded with times being Soil Resource and Development Institute [45]. The dramatic increasing of saline area is caused by rise of the sea levels, global warming.

Wheat is a temperate cultivable cereal crop ranked second after rice mainly cultivated in the north and north-west region of Bangladesh. A huge amount of cultivable lands in the coastal belt remain fallow and the sole cropping pattern is fallow-Aman-fallow. Inclusion of wheat in this traditional saline belt cropping pattern could be an effective means for optimizing land utilization to supplement the food production and nutritional deficit of the ever growing population of Bangladesh. Reclamation of saline soil is very much expensive where salt tolerance wheat genotype selection could be a feasible and cost effective mean for the saline belt.

The soil salinity may responsible for many detrimental effects on plant growth and development at physiological and biochemical level Munns [32]. In saline soils, seeds with lower osmotic potential fail to absorb water; increase the accumulation of toxic ions $\left(\mathrm{Na}^{+}\right.$and $\left.\mathrm{Cl}^{-}\right)$and finally there is a delay, decrease and disruption of seed germination Ashraf and Foolad [8]. Metabolism, physiological act and morphological feature of plant changed by soil salinity in and drastically reduce the growth and yield Ashraf and Harris [5]. Ramaden et al. [35] reported that higher salinity level in the germination media build up a high osmotic pressure in the solution which restricted the uptake of soil water required for proper germination of seed. Seed embryo badly affected by higher salt concentration which result there is a delay and reduction of germination percentage of seed. Percentage of germination, length of coleoptiles, length of 
root and seedling growth reduced by detrimental effect of salinity Lallu and Dixit [26]; Ganndha et al. [19]; Bera et al. [10] and Agnihotri et al. [2]. Cha-um et al. [12] mentioned that plant that was exposed to salinity stress, water potential was reduced then water use efficiency was also reduced. But the salt tolerance species exert a high capacity to resist salt stress through biosynthesis and accumulation of compatible solute. These solutes increase the overall osmotic pressure within the cell there by empowering plant cell to affirm both turgor and driving gradient for water absorption Hasegawa et al. [20]. According to Kumar et al. [25] numerous plants have improved mechanism either to restrict salt uptake to their cell or to tolerate within the cell. Zhu [47] also reported that plant faces salt stress condition; affirm a high $\mathrm{K}^{+}$ concentration and low $\mathrm{Na}^{+}$concentration in the cytosol. They do this by regulation of expression and activity of $\mathrm{K}^{+}$and $\mathrm{Na}^{+}$ transporters and $\mathrm{H}^{+}$pumps that creates the driving force for transport. Regulation of $\mathrm{K}^{+}$uptake and prevention of $\mathrm{Na}^{+}$ influx, promotion of $\mathrm{Na}^{+}$efflux from the cell and use of $\mathrm{Na}^{+}$ for $\mathrm{K}^{+}$adjustment are the strategies generally use by plant to maintain expected $\mathrm{K}^{+} / \mathrm{Na}^{+}$ration in cytosol. So, a high $\mathrm{K}^{+} / \mathrm{Na}^{+}$ratio in cytosol is always expected for normal functioning of the plant cell Zhu [47]. Only physiological markers for example $\mathrm{Na}^{+}, \mathrm{K}^{+}$content, $\mathrm{K}^{+}: \mathrm{Na}^{+}$ratio and accumulation pattern of proline are less suitable, even in the point of view of some researchers are not promising enough for screening the salt tolerance genotypes Shannon [40].

The varietal variation in salinity tolerance that exists among crop plant can be used through screening program by exposing target traits for salt tolerance Kingsbury et al. [24]. Physiological tolerance along with some agronomic traits and their relationship with salt tolerance indices could be a feasible means are considered strong enough to be a selection tool in breeding of salt tolerance cultivars Allakhverdiev et al. [4].

Therefore, with a view to expand the cultivation and to sustain the yield of wheat in the coastal belt, the present piece of work was implemented to evaluate some agronomic and physiological traits of wheat as screening criteria against salinity condition.

\section{Materials and Methods}

The experiment was implemented at the central laboratory of Sher-e-Bangla Agricultural University, Dhaka-1207 from February to March, 2016. The experiment was conducted in single factor Completely Randomize Design (CRD) with five replications.

The performance of 33 wheat genotypes including 5 wheat varieties and 28 advanced lines were collected form Wheat Research Centre, Nashipur, Dinajpur and Bangladesh Agricultural Research Institute, respectively (Table 1) and tested under five different salinity levels $(0,5,10,15$ and 20 $\mathrm{dSm}^{-1}$ ) for present research.
Table 1. List of wheat genotypes used for present research

\begin{tabular}{|c|c|c|}
\hline Sl. No. & Genotypes & Remarks \\
\hline 01 & SATYN-22 & Advanced line \\
\hline 02 & SATYN-15 & Advanced line \\
\hline 03 & SATYN-21 & Advanced line \\
\hline 04 & SAYYN-17 & Advanced line \\
\hline 05 & SATYN-23 & Advanced line \\
\hline 06 & ESWYT-5 & Advanced line \\
\hline 07 & SATYN-24 & Advanced line \\
\hline 08 & ESWYT-6 & Advanced line \\
\hline 09 & SATYN-3 & Advanced line \\
\hline 10 & SATYN-27 & Advanced line \\
\hline 11 & SATYN-12 & Advanced line \\
\hline 12 & SATYN-6 & Advanced line \\
\hline 13 & SATYN-19 & Advanced line \\
\hline 14 & SATYN-16 & Advanced line \\
\hline 15 & SATYN-25 & Advanced line \\
\hline 16 & WICYT-7 & Advanced line \\
\hline 17 & WICYT-9 & Advanced line \\
\hline 18 & WICYT-28 & Advanced line \\
\hline 19 & WICYT-35 & Advanced line \\
\hline 20 & WICYT-41 & Advanced line \\
\hline 21 & WICYT-15 & Advanced line \\
\hline 22 & WICYT-20 & Advanced line \\
\hline 23 & WICYT-25 & Advanced line \\
\hline 24 & WICYT-26 & Advanced line \\
\hline 25 & SATYN-2 & Advanced line \\
\hline 26 & SATYN-10 & Advanced line \\
\hline 27 & SATYN-14 & Advanced line \\
\hline 28 & SATYN-20 & Advanced line \\
\hline 29 & BARI GOM 25 & Variety \\
\hline 30 & BARI GOM 26 & Variety \\
\hline 31 & BARI GOM 27 & Variety \\
\hline 32 & BARI GOM 28 & Variety \\
\hline 33 & BARI GOM 29 & Variety \\
\hline
\end{tabular}

\subsection{Seed Placement for Germination}

33 wheat genotypes were collected from Wheat Research Centre, Nashipur, Dinajpur and Bangladesh Agricultural Research Institute and dried for 3 hrs under sunlight. Filter paper were cut according to the petri dish size and place into the bottom of the dish. 30 seeds of each wheat genotypes were placed into the petri dish. $0,1.4625,2.925,4.3875$ and $5.85 \mathrm{~g} \mathrm{NaCl}$ were dissolved in $500 \mathrm{ml}$ distill water separately to get $0,5,10,15$ and $20 \mathrm{dSm}^{-1} \mathrm{NaCl}$ solution, respectively. The salt solutions were sprayed as per treatment on petri dish until the saturated condition and spraying continued with 6 hrs interval. 


\subsection{Data Recorded on Germination Traits}

Germination was recorded at $24 \mathrm{hrs}$ interval and continued up to $11^{\text {th }}$ day. More than $2 \mathrm{~mm}$ long plumule and radicle seed was considered as germinated seed.

The germination rate was calculated using following formula:

Rate of germination $(\%)=\frac{\text { TotalNumberof germinatedseeds }}{\text { Totalseedplacedforgermination }} \times 100$

Co-efficient of germination (CG) was calculated using the following formula Copeland [14]:

Coefficient of germination $(\%)=\frac{A 1+A 2+\cdots+A x}{A 1 T 1+A 2 T 2+\cdots A x T x} \times 100$

Where,

$A=$ Number of seeds germinated

$\mathrm{T}=$ Time corresponding to $\mathrm{A}$

$\mathrm{x}=$ Number of days to final count

Vigour index was calculated using following formula Abdul-Baki and Anderson [1]:

Vigour index $=\frac{\text { Totalgermination } \times \text { Seedlinglength }(\mathrm{mm})}{100}$

\subsection{Data Recorded on Shoot and Root}

At $11^{\text {th }}$ day after seed placement, five seedlings of each petri dish were randomly sampled. Shoot and root length of single seedling were recorded with meter scale. Then the shoot and root of the seedling were dried for $48 \mathrm{hrs}$ then dry weight of shoot and root were recorded using electric balance.

After recording the fresh weight leaf of each seedling place into petri dish for $24 \mathrm{hrs}$ then leaf soaking with distilled water, then turgid weight was recorded, after $48 \mathrm{hrs}$ drying for the dry weight was measured.

Relative water content was measured using following formula Baque et al. [9]:

Relative water content $\left(\right.$ WRC) $(\%)=\frac{\text { Freshweight }- \text { Dryweight }}{\text { Tugidweight }- \text { Dryweight }}$

$$
\times 100
$$

Water saturation deficit was recorded using following formula Baque et al. [9]:

Water saturation deficit $(\mathrm{WSD})=100$ - Relative water content

Water retention capacity was measured following formula Baque et al. [9]:

Water retention capacity $($ WTC $)=\frac{\text { Turgidweight }}{\text { Dryweight }}$

\subsection{Statistical Analysis}

The collected data were analyzed statistically following CRD design by MSTAT-C computer package program and the treatments were compared by Least Significance Differences (LSD) test.

\section{Result and Discussion}

\subsection{Germination Percentage}

Germination percentage significantly varied among wheat genotypes under different level of saline concentration (Table 2). The rate of germination decreased with the increasing of salt concentration. ESWYT-5, ESWYT-6 and BARI GOM 28 showed consistency against different levels of saline concentration and gave the highest germination rate (98.89, 94.44, 92.11, 91.57 and 88.00\% in ESWYT-5; 97.78, 94.44, 90.19, 90.23 and $87.10 \%$ in ESWYT-6 and 97.46, $92.72,89.89,89.31$ and $86.67 \%$ in BARI GOM 28 at $0,5,10$, 15 and $20 \mathrm{dSm}^{-1}$, respectively). On the other hand the lowest germination rate was counted for BARI GOM 29 and SATYN-20 (32.22 and $33.33 \%$ at $0 \mathrm{dSm}^{-1}$, respectively). The reduction trend of germination percentages rapidly increase with the increasing of $\mathrm{NaCl}$ concentration for BARI GOM 29 $\left(85.00,87.78,88.89\right.$ and 90.00 at $5,10,15$ and $20 \mathrm{dSm}^{-1}$, respectively). So, in the context of germination percentage to salinity tolerance/sensitivity, it may be concluded that among 33 wheat genotypes ESWYT-5, ESWYT-6 and BARI GOM 28 might be referred as salt tolerance cultivars and BARI GOM 29 as most salinity sensitive cultivar. The germination of wheat genotype could be affected in two ways under salinity condition. First of all, in the germination media excess salt might be reduced the osmotic potential of tested seed to such extent that seeds that were placed for germination unable to absorb enough water necessary for transportation of mineral nutrients which were crucial for germination and the second one was is the embryo of the tested seed adversely affected by the toxicity of salt salutes Mujeeb-ur-Rahman et al. [31]. In the physiological point of view, the absorption of more $\mathrm{K}^{+} / \mathrm{Na}^{+}$is essential for seed germination. The rise of salinity level decreases the $\mathrm{K}^{+} / \mathrm{Na}^{+}$ ratio Carmer et al. [15] which injures the embryo of the germinal seed. Khan et al. [22] also reported that, up taking of salt in sensitive plant competes with the up taking of compatible nutrients ions, especially $\mathrm{K}^{+}$, which causes $\mathrm{K}^{+}$ deficiency. In salinity stress condition, sensitive wheat genotypes absorb more $\mathrm{Na}^{+}$than $\mathrm{K}^{+}$Ashraf and Oleary [7] and Sairamet al. [37] as a result germination decreases. Amount of $\mathrm{Na}^{+}$uptake by cereals was reported as salt tolerant indices Ashraf and Khanum [6]. In some halophytes like wheat, the exclusion of $\mathrm{Na}^{+}$and inclusion of $\mathrm{K}^{+}$, salt tolerance mechanism might absence referred as sensitive cultivar Poustini and Siosemardeh [33]. On the other hand increasing the absorption of osmotically active constituents like sugar organic acid, proline, glycine, $\mathrm{K}^{+}$and $\mathrm{Cl}^{-}$which trigger the nutrient release selectivity and osmotic adjustment to salinity referred as salt tolerance genotypes. Similar result also found by different scientists in different crops like: Khatkar and Kuhad [23] in wheat; Shirazi [42], Lallu and Dixit [26] in mustard; Bera et al. [10] in chickpea. 
Table 2. Effect of salinity level on germination rate of different wheat genotypes at different salt concentrations

\begin{tabular}{|c|c|c|c|c|c|}
\hline \multirow{3}{*}{$\begin{array}{l}\text { Genotypes } \\
\text { SATYN-22 }\end{array}$} & \multicolumn{5}{|c|}{ Germination rate (\%) at different salt concentrations } \\
\hline & $0 \mathrm{dS} \mathrm{m}^{-1}$ & $5 \mathrm{dS} \mathrm{m}^{-1}$ & $10 \mathrm{dS} \mathrm{m}^{-1}$ & $15 \mathrm{dS} \mathrm{m}^{-1}$ & $20 \mathrm{dS} \mathrm{m}^{-1}$ \\
\hline & $77.33 \quad \mathrm{i}$ & 75.55 f-h & $51.11 \mathrm{n}$ & $41.10 \mathrm{kl}$ & $33.34 \mathrm{k} 1$ \\
\hline SATYN-15 & 88.89 e-g & 80.00 de & 73.44 f-h & $67.05 \mathrm{e}$ & 53.31 ef \\
\hline SATYN-21 & 88.89 e-g & $87.78 \mathrm{c}$ & $82.40 \quad \mathrm{bc}$ & 66.66 ef & $63.60 \mathrm{~d}$ \\
\hline SAYYN-17 & $77.66 \quad \mathrm{i}$ & $52.22 \quad 1$ & $48.05 \mathrm{n}$ & $43.14 \mathrm{k}$ & $31.11 \mathrm{~lm}$ \\
\hline SATYN-23 & $87.78 \mathrm{f}-\mathrm{h}$ & 74.44 g-i & $66.66 \mathrm{jk}$ & $67.77 \mathrm{e}$ & $55.55 \mathrm{e}$ \\
\hline ESWYT-5 & 98.89 a & $94.44 \quad \mathrm{a}$ & $92.11 \mathrm{a}$ & $91.57 \mathrm{a}$ & 88.00 a \\
\hline SATYN-24 & $84.44 \mathrm{~h}$ & $82.11 \mathrm{~d}$ & 74.44 e-g & $75.55 \mathrm{c}$ & $67.77 \mathrm{c}$ \\
\hline ESWYT-6 & $97.78 \mathrm{ab}$ & $94.44 \quad \mathrm{a}$ & 90.19 a & $90.23 \quad \mathrm{a}$ & $87.10 \quad \mathrm{a}$ \\
\hline SATYN-3 & $62.22 \mathrm{k}$ & $50.00 \quad 1$ & $31.11 \mathrm{o}$ & $30.22 \mathrm{~m}$ & $25.55 \mathrm{n}$ \\
\hline SATYN-27 & $76.66 \quad \mathrm{i}$ & $65.55 \mathrm{k}$ & $47.75 \mathrm{n}$ & $38.47 \quad 1$ & $35.98 \quad \mathrm{jk}$ \\
\hline SATYN-12 & $70.00 \quad \mathrm{j}$ & $68.92 \mathrm{jk}$ & $62.22 \mathrm{~lm}$ & $50.00 \mathrm{j}$ & $37.77 \quad \mathrm{j}$ \\
\hline SATYN-6 & $93.33 \mathrm{~cd}$ & 88.89 bc & 84.49 b & $76.66 \mathrm{c}$ & $71.44 \quad b$ \\
\hline SATYN-19 & 88.89 e-g & 78.89 d-f & 74.44 e-g & 66.66 ef & $45.55 \quad \mathrm{i}$ \\
\hline SATYN-16 & $86.66 \mathrm{gh}$ & 78.89 d-f & $64.44 \mathrm{kl}$ & $63.33 \mathrm{f}-\mathrm{h}$ & $49.96 \mathrm{gh}$ \\
\hline SATYN-25 & 91.11 d-f & $82.22 \mathrm{~d}$ & $78.88 \mathrm{~cd}$ & $73.86 \mathrm{~cd}$ & $68.89 \mathrm{bc}$ \\
\hline WICYT-7 & $93.33 \mathrm{~cd}$ & $70.00 \quad \mathrm{j}$ & $61.11 \mathrm{~lm}$ & $59.51 \quad \mathrm{i}$ & $55.55 \mathrm{e}$ \\
\hline WICYT-9 & $88.88 \quad$ e-g & $82.22 \mathrm{~d}$ & $68.89 \mathrm{ij}$ & $62.89 \mathrm{~g}-\mathrm{i}$ & $52.17 \mathrm{fg}$ \\
\hline WICYT-28 & $93.33 \mathrm{~cd}$ & 75.55 f-h & 70.51 h-j & $66.43 \quad \mathrm{e}-\mathrm{g}$ & 54.98 ef \\
\hline WICYT-35 & $91.11 \mathrm{~d}-\mathrm{f}$ & $82.22 \mathrm{~d}$ & $75.55 \mathrm{def}$ & $67.77 \mathrm{e}$ & $62.03 \mathrm{~d}$ \\
\hline WICYT-41 & $94.42 \quad b-d$ & $77.77 \quad$ e-g & 74.44 efg & $71.63 \mathrm{~d}$ & $68.62 \mathrm{bc}$ \\
\hline WICYT-15 & $80.00 \quad \mathrm{i}$ & $71.11 \quad \mathrm{ij}$ & $60.00 \mathrm{~m}$ & $61.11 \mathrm{hi}$ & $61.82 \mathrm{~d}$ \\
\hline WICYT-20 & $94.44 \quad b-d$ & $65.55 \mathrm{k}$ & 63.33 k-m & 60.15 hi & $56.22 \mathrm{e}$ \\
\hline WICYT-25 & $48.88 \quad 1$ & $34.39 \mathrm{~m}$ & $31.24 \quad \mathrm{o}$ & $15.55 \quad$ o & $10.37 \mathrm{p}$ \\
\hline WICYT-26 & $92.22 \quad \mathrm{c}-\mathrm{e}$ & $77.77 \quad$ e-g & 76.66 d-f & $67.77 \mathrm{e}$ & $48.89 \mathrm{~h}$ \\
\hline SATYN-2 & $95.55 \quad \mathrm{a}-\mathrm{c}$ & $87.77 \quad \mathrm{c}$ & $85.55 \mathrm{~b}$ & $84.44 \quad \mathrm{~b}$ & $67.77 \mathrm{c}$ \\
\hline SATYN-10 & $95.55 \quad a-c$ & $88.88 \quad$ bc & $77.77 \mathrm{de}$ & $75.55 \mathrm{c}$ & $66.66 \mathrm{c}$ \\
\hline SATYN-14 & $78.89 \quad \mathrm{i}$ & $72.22 \quad h-j$ & 70.59 g-i & $51.11 \quad \mathrm{j}$ & $49.64 \mathrm{gh}$ \\
\hline SATYN-20 & $33.33 \mathrm{n}$ & $28.88 \mathrm{n}$ & $17.77 \quad \mathrm{q}$ & 14.74 ор & $9.988 \mathrm{p}$ \\
\hline BARI GOM 25 & $37.77 \quad \mathrm{~m}$ & $34.83 \mathrm{~m}$ & $24.44 \mathrm{p}$ & $19.94 \mathrm{n}$ & 19.98 о \\
\hline BARI GOM 26 & $92.22 \quad \mathrm{c}-\mathrm{e}$ & $77.77 \quad$ e-g & 63.33 k-m & $61.11 \mathrm{hi}$ & 53.42 ef \\
\hline BARI GOM 27 & $84.44 \mathrm{~h}$ & $53.33 \quad 1$ & $31.11 \quad \mathrm{o}$ & $27.77 \mathrm{~m}$ & $28.89 \mathrm{~m}$ \\
\hline BARI GOM 28 & $97.46 \quad \mathrm{ab}$ & $92.72 \mathrm{ab}$ & 89.89 a & 89.31 a & 86.67 a \\
\hline BARI GOM 29 & $32.22 \mathrm{n}$ & $15.00 \quad \mathrm{o}$ & $12.22 \mathrm{r}$ & $11.11 \mathrm{p}$ & $10.00 \mathrm{p}$ \\
\hline $\operatorname{LSD}_{(0.05)}$ & 3.66 & 4.12 & 3.91 & 3.67 & 3.03 \\
\hline CV (\%) & 3.58 & 4.64 & 4.97 & 5.06 & 4.77 \\
\hline
\end{tabular}

Values having same letter(s) do not differed significantly by least significant difference (LSD) at $5 \%$ level

\subsection{Shoot Length}

Salinity level significantly affected the shoot length of wheat genotypes (Table 3). The magnitude of reduction of shoot length was lower in ESWYT-5, ESWYT-6 and BARI GOM 28 under different levels of salt concentration. The shoot length ranged from $180.2 \mathrm{~mm}$ in ESWYT-5 to 122.00 in BARI GOM 29 at control solution $\left(0 \mathrm{dSm}^{-1}\right) ; 161.70 \mathrm{~mm}$ in ESWYT-5 to $39.60 \mathrm{~mm}$ in SATYN-20 at $5 \mathrm{dSm}^{-1} \mathrm{NaCl}$ solution; $147.20 \mathrm{~mm}$ in ESWYT-5 to $28.27 \mathrm{~mm}$ in SATYN-14 at $10 \mathrm{dSm}^{-1} \mathrm{NaCl}$ solution; $120.50 \mathrm{~mm}$ in ESWYT-5 to $10.47 \mathrm{~mm}$ in WICYT-41 at $15 \mathrm{dSm}^{-1} \mathrm{NaCl}$ solution. Wheat genotype ESWYT-5 showed statistically similarity with ESWYT-6 at $5 \mathrm{dSm}^{-1} \mathrm{NaCl}$ solution; at 15 $\mathrm{dSm}^{-1} \mathrm{NaCl}$ concentrations, WICYT- 41 showed statistically at par with WICYT-9 and WICYT-14 wheat genotypes. At $20 \mathrm{dSm}^{-1} \mathrm{NaCl}$ solution maximum shoot length was recorded for ESWYT-5 followed by ESWYT-6 and BARI GOM 28 
but there was a dramatic change of shoot length in SATYN-22 and SATYN-25 and they did not survive at 20 $\mathrm{dSm}^{-1} \mathrm{NaCl}$ solution. The lower shoot length for salt sensitive cultivars might be due to more accumulation of $\mathrm{Na}^{+}$ which retard the cell division and elongation process and ultimately reduce the shoot length of salt sensitive cultivars For a consequence it might be concluded that ESWYT-5, ESWYT-6 and BARI GOM 28 wheat cultivars may be tolerance to salt.

Table 3. Effect of salinity level on shoot length of different wheat genotypes at different salt concentrations

\begin{tabular}{|c|c|c|c|c|c|}
\hline \multirow{3}{*}{$\begin{array}{l}\text { Genotypes } \\
\text { SATYN-22 }\end{array}$} & \multicolumn{5}{|c|}{ Shoot length $(\mathrm{mm})$ at different salt concentrations } \\
\hline & $0 \mathrm{dS} \mathrm{m}^{-1}$ & $5 \mathrm{dS} \mathrm{m}^{-1}$ & $10 \mathrm{dS} \mathrm{m}^{-1}$ & $15 \mathrm{dS} \mathrm{m}^{-1}$ & $20 \mathrm{dS} \mathrm{m}^{-1}$ \\
\hline & 147.3 c-e & $140.0 \quad \mathrm{c}-\mathrm{e}$ & 40.35 o & 33.35 p-r & 0.00 \\
\hline SATYN-15 & $136.4 \mathrm{~g}-\mathrm{k}$ & $129.0 \mathrm{fg}$ & $59.13 \mathrm{~m}$ & $41.79 \mathrm{n}$ & $29.03 \mathrm{k}$ \\
\hline SATYN-21 & $131.8 \mathrm{j}-\mathrm{n}$ & $128.5 \mathrm{fg}$ & $103.5 \mathrm{~d}$ & $91.13 \mathrm{c}$ & 39.53 hi \\
\hline SAYYN-17 & $134.1 \mathrm{~h}-1$ & $125.0 \quad \mathrm{~g}-\mathrm{j}$ & 100.7 de & $68.60 \mathrm{~h}$ & $37.58 \quad \mathrm{ij}$ \\
\hline SATYN-23 & $139.3 \mathrm{f}-\mathrm{j}$ & $114.5 \quad \mathrm{k}-\mathrm{m}$ & 98.15 ef & $81.36 \mathrm{f}$ & $29.31 \mathrm{k}$ \\
\hline ESWYT-5 & 180.2 a & 161.7 a & $147.2 \mathrm{a}$ & 120.5 a & $116.2 \quad \mathrm{a}$ \\
\hline SATYN-24 & 130.7 k-n & $117.3 \quad \mathrm{k}-\mathrm{m}$ & $101.5 \mathrm{de}$ & $86.17 \mathrm{de}$ & $71.40 \mathrm{~d}$ \\
\hline ESWYT-6 & $169.8 \mathrm{~b}$ & $157.3 \mathrm{ab}$ & $138.7 \mathrm{~b}$ & $106.6 \mathrm{~b}$ & $99.72 \quad b$ \\
\hline SATYN-3 & $152.9 \mathrm{c}$ & $140.7 \mathrm{~cd}$ & 83.96 ij & $45.87 \mathrm{~m}$ & $37.87 \quad \mathrm{ij}$ \\
\hline SATYN-27 & 124.3 no & $120.6 \mathrm{~h}-\mathrm{k}$ & $57.70 \mathrm{~m}$ & $55.73 \mathrm{j}$ & $49.20 \mathrm{f}$ \\
\hline SATYN-12 & 140.1 e-i & 133.9 ef & $93.87 \mathrm{fg}$ & $89.26 \mathrm{~cd}$ & $82.33 \mathrm{c}$ \\
\hline SATYN-6 & 140.7 e-h & $105.5 \quad \mathrm{o}$ & 87.27 h-j & 84.71 ef & $82.14 \quad \mathrm{c}$ \\
\hline SATYN-19 & 127.9 1-o & $111.3 \quad \mathrm{~m}-\mathrm{o}$ & 86.90 h-j & $53.07 \mathrm{jk}$ & $38.53 \quad \mathrm{i}$ \\
\hline SATYN-16 & $136.3 \mathrm{~g}-\mathrm{k}$ & $112.4 \quad 1-\mathrm{n}$ & $77.20 \mathrm{k}$ & $70.58 \mathrm{gh}$ & $45.73 \mathrm{~g}$ \\
\hline SATYN-25 & 132.9 h-m & 133.6 ef & 98.00 ef & $73.92 \mathrm{~g}$ & 0.00 \\
\hline WICYT-7 & 136.7 g-k & $70.00 \quad \mathrm{q}$ & $57.49 \mathrm{~m}$ & 38.13 no & $9.068 \mathrm{q}$ \\
\hline WICYT-9 & $139.1 \mathrm{f}-\mathrm{j}$ & 50.60 & $31.00 \mathrm{rst}$ & $11.07 \mathrm{v}$ & $8.836 \mathrm{q}$ \\
\hline WICYT-28 & 129.7 k-o & $50.83 \quad \mathrm{r}$ & 38.87 op & 30.42 q-s & $13.07 \quad n-p$ \\
\hline WICYT-35 & 143.6 d-g & $114.4 \quad \mathrm{k}-\mathrm{m}$ & $33.23 \mathrm{q}-\mathrm{s}$ & $24.27 \mathrm{t}$ & $15.47 \mathrm{n}$ \\
\hline WICYT-41 & 142.2 d-g & $126.7 \mathrm{gh}$ & $45.67 \mathrm{n}$ & $10.47 \mathrm{v}$ & $9.928 \quad \mathrm{q}$ \\
\hline WICYT-15 & $143.3 \mathrm{~d}-\mathrm{g}$ & $70.51 \quad \mathrm{q}$ & $65.73 \quad 1$ & $51.27 \mathrm{kl}$ & $42.20 \mathrm{~h}$ \\
\hline WICYT-20 & $132.7 \quad \mathrm{i}-\mathrm{m}$ & $48.87 \quad \mathrm{r}$ & $35.67 \mathrm{pq}$ & 27.37 st & $21.60 \mathrm{~m}$ \\
\hline WICYT-25 & $132.8 \quad \mathrm{i}-\mathrm{m}$ & $126.7 \mathrm{gh}$ & $56.40 \mathrm{~m}$ & 36.75 op & $24.85 \quad 1$ \\
\hline WICYT-26 & $153.2 \mathrm{c}$ & $142.5 \mathrm{~cd}$ & 41.20 no & 36.73 op & $26.66 \mathrm{kl}$ \\
\hline SATYN-2 & $146.1 \quad c-f$ & $136.6 \mathrm{de}$ & $82.87 \mathrm{j}$ & $73.00 \mathrm{~g}$ & $13.27 \quad n-p$ \\
\hline SATYN-10 & 127.1 1-o & $85.80 \mathrm{p}$ & 35.03 p-r & $34.07 \mathrm{pq}$ & $28.17 \mathrm{k}$ \\
\hline SATYN-14 & 124.3 no & 107.7 no & $28.27 \mathrm{t}$ & $12.53 \mathrm{v}$ & 13.80 no \\
\hline SATYN-20 & 129.1 k-o & $39.60 \mathrm{~s}$ & 28.80 st & $18.14 \mathrm{u}$ & $11.40 \quad \mathrm{o}-\mathrm{q}$ \\
\hline BARI GOM 25 & $149.9 \mathrm{~cd}$ & $143.6 \mathrm{c}$ & $64.00 \quad 1$ & $60.47 \mathrm{i}$ & $55.61 \mathrm{e}$ \\
\hline BARI GOM 26 & $125.6 \mathrm{~m}-\mathrm{o}$ & $118.8 \quad \mathrm{j}-1$ & $87.63 \mathrm{hi}$ & $55.47 \mathrm{j}$ & $50.07 \mathrm{f}$ \\
\hline BARI GOM 27 & $129.2 \mathrm{k}-\mathrm{o}$ & 126.1 g-i & $89.47 \mathrm{gh}$ & $49.20 \mathrm{~lm}$ & $35.00 \quad \mathrm{j}$ \\
\hline BARI GOM 28 & $167.4 \mathrm{~b}$ & $153.2 \quad b$ & $134.0 \mathrm{c}$ & $104.7 \quad \mathrm{~b}$ & $97.17 \quad b$ \\
\hline BARI GOM 29 & $122.0 \quad$ o & $119.8 \quad \mathrm{i}-\mathrm{k}$ & 33.70 qr & 29.54 rs & $10.47 \mathrm{pq}$ \\
\hline $\operatorname{LSD}_{(0.05)}$ & 7.90 & 6.43 & 4.59 & 3.84 & 2.99 \\
\hline CV (\%) & 4.53 & 4.51 & 5.12 & 5.61 & 6.34 \\
\hline
\end{tabular}

Values having same letter(s) do not differed significantly by least significant difference (LSD) at $5 \%$ level 


\subsection{Root Length}

The root length was varied significantly among wheat genotypes under different salt concentration (Table 4). Consistently ESWYT-5, ESWYT-6 and BARI GOM 28 wheat genotypes had shown a slower reduction against the increasing of salt concentration. At $0 \mathrm{dSm}^{-1}$ salt concentration root length ranged from $164.20 \mathrm{~mm}$ in ESWYT-5 to $13.32 \mathrm{~mm}$ in SATYN-12; at 5, 10 and $15 \mathrm{dSm}^{-1}$ salt concentrations root length ranges from $152.10 \mathrm{~mm}$ in ESWYT-5 to $5.13 \mathrm{~mm}$ in SATYN-19, $109.30 \mathrm{~mm}$ in ESWYT-5 to $3.96 \mathrm{~mm}$ in SATYN-19, $69.79 \mathrm{~mm}$ in ESWYT-5 to $3.03 \mathrm{~mm}$ in SATYN-19, respectively. Longest root length distinctly was found from ESWYT-5, ESWYT-6 and BARI GOM 28 at $20 \mathrm{dSm}^{-1}$, on the other hand seedlings of SATYN-22 and SATYN-25 did not survive at the same salt concentration. In conclusion, it may be said that
ESWYT-5, ESWYT-6 and BARI GOM 28 wheat genotypes could be salt tolerance and SATYN-19, SATYN-12 sensitive to saline condition in respect of root length. SATYN-22 and SATYN-25 might be very much sensitive to salt at higher salt concentration in the context of root length. Shoot and root length severely affected by salt stress and as a consequence there was a drastic reduction observed for salt stress sensitive genotypes. It has been found that, under slat stress condition photosynthetic rate is reduced markedly, lost huge energy in salt removal mechanism, reduced transportation of compatible nutrient, arrested cell division and enlargement, decreased shoot length, for consequence there was a marked reduction of shoot and root length. Similar findings also reported by Moud and Maghsoudi [30], Datta et al. [17], Mujeeb et al. [31], Tarmatt and Munns [46] and Dager et al. [16].

Table 4. Effect of salinity level on root length of different wheat genotypes at different salt concentrations

\begin{tabular}{|c|c|c|c|c|c|c|c|c|c|}
\hline \multirow{3}{*}{$\begin{array}{c}\text { Genotypes } \\
\text { SATYN-22 } \\
\end{array}$} & \multicolumn{9}{|c|}{ Root length (mm) at different salt concentrations } \\
\hline & $0 \mathrm{dS} \mathrm{m}^{-1}$ & \multicolumn{2}{|c|}{$5 \mathrm{dS} \mathrm{m}^{-1}$} & \multicolumn{2}{|c|}{$10 \mathrm{dS} \mathrm{m}^{-1}$} & \multicolumn{2}{|c|}{$15 \mathrm{dS} \mathrm{m}^{-1}$} & \multicolumn{2}{|c|}{$20 \mathrm{dS} \mathrm{m}^{-1}$} \\
\hline & $129.1 \mathrm{~d}$ & 87.67 & g-i & 36.23 & 1 & 27.69 & $\mathrm{o}$ & 0.00 & $\mathrm{~s}$ \\
\hline SATYN-15 & 120.5 ef & 84.47 & $\mathrm{ij}$ & 42.41 & $\mathrm{ij}$ & 50.85 & $\mathrm{~g}$ & 34.20 & $\mathrm{e}$ \\
\hline SATYN-21 & $119.6 \mathrm{f}$ & 90.40 & $\mathrm{f}-\mathrm{h}$ & 65.87 & $\mathrm{e}$ & 58.71 & $\mathrm{e}$ & 27.33 & $\mathrm{~g}$ \\
\hline SAYYN-17 & $113.1 \mathrm{gh}$ & 109.2 & $\mathrm{~d}$ & 62.87 & $\mathrm{f}$ & 53.30 & fg & 41.84 & $\mathrm{~d}$ \\
\hline SATYN-23 & 142.0 & 92.93 & $\mathrm{f}$ & 65.64 & $\mathrm{e}$ & 64.57 & $\mathrm{bc}$ & 28.96 & $\mathrm{f}$ \\
\hline ESWYT-5 & 164.2 & 152.1 & $\mathrm{a}$ & 109.3 & $\mathrm{a}$ & 69.79 & $\mathrm{a}$ & 64.80 & $\mathrm{a}$ \\
\hline SATYN-24 & 82.03 no & 120.5 & $\mathrm{c}$ & 65.07 & ef & 63.37 & $\mathrm{bc}$ & 23.73 & ijk \\
\hline ESWYT-6 & $160.29 \quad \mathrm{a}$ & 150.1 & $\mathrm{a}$ & 95.48 & $\mathrm{~b}$ & 65.13 & $\mathrm{~b}$ & 63.72 & $\mathrm{a}$ \\
\hline SATYN-3 & $115.7 \mathrm{fg}$ & 72.57 & 1 & 64.35 & ef & 28.09 & no & 29.42 & $\mathrm{f}$ \\
\hline SATYN-27 & $89.13 \quad \mathrm{~lm}$ & 82.89 & j & 24.83 & $\mathrm{o}$ & 31.60 & $\operatorname{lm}$ & 18.80 & $\mathrm{~m}$ \\
\hline SATYN-12 & $13.32 \mathrm{p}$ & 7.83 & rs & 16.99 & $\mathrm{q}$ & 5.048 & $\mathrm{~s}$ & 5.91 & $\mathrm{q}$ \\
\hline SATYN-6 & $114.8 \quad \mathrm{fg}$ & 91.67 & $\mathrm{fg}$ & 44.07 & $\mathrm{i}$ & 51.00 & $\mathrm{~g}$ & 43.67 & $\mathrm{c}$ \\
\hline SATYN-19 & $14.99 \quad \mathrm{p}$ & 5.13 & $\mathrm{~s}$ & 3.96 & $\mathrm{~s}$ & 3.03 & $\mathrm{~s}$ & 4.21 & $\mathrm{r}$ \\
\hline SATYN-16 & 120.4 ef & 107.6 & $\mathrm{~d}$ & 79.40 & $\mathrm{c}$ & 60.07 & $\mathrm{de}$ & 51.33 & $\mathrm{~b}$ \\
\hline SATYN-25 & $121.1 \mathrm{gh}$ & 86.60 & $h-j$ & 56.27 & $\mathrm{~g}$ & 62.13 & $\mathrm{~cd}$ & 0.00 & $\mathrm{~s}$ \\
\hline WICYT-7 & $85.33 \mathrm{mn}$ & 25.07 & 0 & 13.27 & $\mathrm{r}$ & 32.80 & $\mathrm{kl}$ & 16.73 & $\mathrm{n}$ \\
\hline WICYT-9 & $130.8 \quad \mathrm{~d}$ & 14.33 & $\mathrm{p}$ & 21.27 & $\mathrm{p}$ & 30.20 & $\mathrm{mn}$ & 26.00 & gh \\
\hline WICYT-28 & $91.07 \quad \mathrm{kl}$ & 9.83 & $\mathrm{qr}$ & 41.80 & ijk & 24.87 & $\mathrm{p}$ & 19.07 & $\mathrm{~m}$ \\
\hline WICYT-35 & $95.00 \quad \mathrm{jk}$ & 14.07 & $\mathrm{pq}$ & 29.67 & $\mathrm{n}$ & 43.30 & $\mathrm{i}$ & 25.07 & hi \\
\hline WICYT-41 & $105.5 \quad \mathrm{i}$ & 32.27 & $\mathrm{n}$ & 23.27 & op & 33.73 & $\mathrm{kl}$ & 14.80 & o \\
\hline WICYT-15 & $116.4 \mathrm{fg}$ & 35.87 & $\mathrm{n}$ & 58.47 & $\mathrm{~g}$ & 34.80 & $\mathrm{k}$ & 34.53 & $\mathrm{e}$ \\
\hline WICYT-20 & $112.5 \mathrm{gh}$ & 23.53 & o & 70.87 & $\mathrm{~d}$ & 54.87 & $\mathrm{f}$ & 34.47 & $\mathrm{e}$ \\
\hline WICYT-25 & $113.3 \mathrm{gh}$ & 90.67 & $\mathrm{f}-\mathrm{h}$ & 52.28 & $\mathrm{~h}$ & 48.15 & $\mathrm{~h}$ & 41.04 & $\mathrm{~d}$ \\
\hline WICYT-26 & $107.7 \quad$ hi & 64.00 & $\mathrm{~m}$ & 41.47 & $\mathrm{jk}$ & 18.10 & $\mathrm{q}$ & 9.096 & $\mathrm{p}$ \\
\hline SATYN-2 & $128.2 \mathrm{~d}$ & 67.60 & $\mathrm{~m}$ & 42.90 & $\mathrm{ij}$ & 19.65 & $\mathrm{q}$ & 22.93 & $\mathrm{jkl}$ \\
\hline SATYN-10 & $99.27 \quad \mathrm{j}$ & 63.67 & $\mathrm{~m}$ & 33.80 & $1 \mathrm{~m}$ & 40.60 & $\mathrm{j}$ & 24.20 & $\mathrm{ij}$ \\
\hline SATYN-14 & $126.7 \mathrm{~d}$ & 74.00 & $\mathrm{kl}$ & 35.47 & 1 & 20.00 & $\mathrm{q}$ & 26.53 & $\mathrm{gh}$ \\
\hline SATYN-20 & $77.53 \quad$ o & 18.07 & $\mathrm{p}$ & 14.28 & $\mathrm{r}$ & 10.07 & $\mathrm{r}$ & 6.520 & $\mathrm{q}$ \\
\hline BARI GOM 25 & $91.13 \quad \mathrm{kl}$ & 77.20 & $\mathrm{k}$ & 32.17 & $\mathrm{~m}$ & 28.40 & no & 22.50 & $\mathrm{kl}$ \\
\hline BARI GOM 26 & $128.3 \quad \mathrm{~d}$ & 99.47 & $\mathrm{e}$ & 58.40 & $\mathrm{~g}$ & 24.60 & $\mathrm{p}$ & 22.88 & $\mathrm{jkl}$ \\
\hline BARI GOM 27 & $125.3 \mathrm{de}$ & 82.40 & $\mathrm{j}$ & 39.53 & $\mathrm{k}$ & 26.07 & op & 22.08 & 1 \\
\hline BARI GOM 28 & $150.9 \quad \mathrm{~b}$ & 138.3 & $\mathrm{~b}$ & 94.6 & $\mathrm{~b}$ & 68.59 & $\mathrm{a}$ & 63.68 & $\mathrm{a}$ \\
\hline BARI GOM 29 & $138.3 \mathrm{c}$ & 94.56 & $\mathrm{f}$ & 25.15 & $\mathrm{o}$ & 20.33 & $\mathrm{q}$ & 6.420 & $\mathrm{q}$ \\
\hline $\operatorname{LSD}_{(0.05)}$ & 5.66 & 4.45 & & 2.5 & & 2.4 & & 1.5 & \\
\hline CV (\%) & 4.18 & 4.96 & & $4.2^{\prime}$ & & 5.2 & & 4.8 & \\
\hline
\end{tabular}

Values having same letter(s) do not differed significantly by least significant difference (LSD) at $5 \%$ level 


\subsection{Shoot Dry Weight}

Salinity level had highly significant influence on shoot dry weight of different wheat genotypes (Table 5). ESWYT-5, ESWYT-6 and BARI GOM 28 wheat genotypes showed consistently slower reduction for shoot dry weight with the increasing of salinity levels. Maximum shoot dry weight was reported from ESWYT-5 genotypes followed by ESWYT-6 and BARI GOM 28 at all the $\mathrm{NaCl}$ concentrations whereas
SATYN-22 and SATYN-12 wheat genotypes showed more sensitivity to salinity stress condition and produced lowest shoot dry weight. At $20 \mathrm{dSm}^{-1}$ salt concentration, SATYN-22 and SATYN-12 wheat genotypes did not survive. Therefore, ESWYT-5, ESWYT-6 and BARI GOM 28 wheat genotypes showed promising performance against salinity stress condition in terms of shoot dry weight.

Table 5. Effect of salinity level on shoot dry weight of different wheat genotypes at different salt concentrations

\begin{tabular}{|c|c|c|c|c|c|}
\hline \multirow{2}{*}{ Genotypes } & \multicolumn{5}{|c|}{ Shoot dry weight $(\mathrm{mg})$ at different salt concentrations } \\
\hline & $0 \mathrm{dS} \mathrm{m}^{-1}$ & $5 \mathrm{dS} \mathrm{m}^{-1}$ & $10 \mathrm{dS} \mathrm{m}^{-1}$ & $15 \mathrm{dS} \mathrm{m}^{-1}$ & $20 \mathrm{dS} \mathrm{m}^{-1}$ \\
\hline SATYN-22 & $6.42 \mathrm{p}$ & $5.44 \mathrm{q}$ & $3.65 \mathrm{t}$ & $2.54 \quad \mathrm{o}$ & $0.00 \mathrm{w}$ \\
\hline SATYN-15 & $7.46 \quad 1-n$ & $7.26 \mathrm{mn}$ & $6.49 \quad$ o & $3.76 \mathrm{~m}$ & $3.11 \mathrm{rs}$ \\
\hline SATYN-21 & 7.89 k-m & $7.42 \mathrm{~lm}$ & $7.51 \quad \mathrm{i}-\mathrm{k}$ & $6.72 \mathrm{f}$ & $5.17 \mathrm{i}-\mathrm{k}$ \\
\hline SAYYN-17 & $7.97 \quad \mathrm{kl}$ & $7.55 \mathrm{~lm}$ & $7.57 \quad h-j$ & $6.83 \mathrm{f}$ & $4.90 \quad \mathrm{j}-\mathrm{m}$ \\
\hline SATYN-23 & $7.55 \operatorname{lmn}$ & 6.64 op & 4.92 rs & $3.98 \mathrm{~m}$ & 3.53 p-r \\
\hline ESWYT-5 & $16.64 \quad \mathrm{a}$ & $13.90 \quad \mathrm{a}$ & $13.07 \mathrm{a}$ & $11.30 \mathrm{a}$ & $9.18 \mathrm{a}$ \\
\hline SATYN-24 & $6.60 \quad$ op & $5.31 \mathrm{qr}$ & $5.11 \mathrm{qr}$ & $6.78 \mathrm{f}$ & $6.50 \mathrm{de}$ \\
\hline ESWYT-6 & $15.73 \quad \mathrm{~b}$ & $13.25 \mathrm{~b}$ & $12.46 \mathrm{~b}$ & $10.46 \mathrm{~b}$ & $8.53 \mathrm{~b}$ \\
\hline SATYN-3 & $9.43 \quad \mathrm{i}$ & $7.33 \mathrm{mn}$ & $5.28 \mathrm{qr}$ & $5.23 \mathrm{k}$ & $4.47 \mathrm{mn}$ \\
\hline SATYN-27 & $7.35 \mathrm{mn}$ & $6.74 \quad \mathrm{o}$ & $6.53 \quad 0$ & $5.56 \quad \mathrm{ij}$ & $4.76 \mathrm{k}-\mathrm{m}$ \\
\hline SATYN-12 & $6.03 \mathrm{p}$ & $3.12 \mathrm{t}$ & $3.62 \mathrm{t}$ & $2.62 \quad 0$ & $2.28 \mathrm{uv}$ \\
\hline SATYN-6 & $7.70 \quad \mathrm{~lm}$ & 6.31 op & 7.95 e-h & $7.80 \quad \mathrm{e}$ & 6.31 ef \\
\hline SATYN-19 & $7.87 \quad \mathrm{k}-\mathrm{m}$ & $6.21 \mathrm{p}$ & $7.33 \quad \mathrm{j}-1$ & $6.91 \mathrm{f}$ & 6.28 ef \\
\hline SATYN-16 & $8.37 \quad \mathrm{jk}$ & $8.13 \mathrm{jk}$ & 8.15 ef & $6.71 \mathrm{f}$ & $4.81 \quad \mathrm{j}-\mathrm{m}$ \\
\hline SATYN-25 & $7.50 \quad 1-\mathrm{n}$ & $7.55 \mathrm{~lm}$ & $6.78 \mathrm{~m}-\mathrm{o}$ & $5.71 \quad$ hi & $0.00 \quad \mathrm{w}$ \\
\hline WICYT-7 & 13.47 ef & 10.98 ef & $8.30 \mathrm{e}$ & $8.12 \mathrm{~d}$ & $6.93 \mathrm{~cd}$ \\
\hline WICYT-9 & $11.85 \mathrm{gh}$ & $9.92 \mathrm{~g}$ & $7.11 \mathrm{k}-\mathrm{m}$ & $5.35 \mathrm{jk}$ & $2.62 \mathrm{tu}$ \\
\hline WICYT-28 & $11.46 \mathrm{~h}$ & $3.81 \quad \mathrm{~s}$ & $3.43 \mathrm{t}$ & $3.15 \mathrm{n}$ & $3.37 \mathrm{q}-\mathrm{s}$ \\
\hline WICYT-35 & 7.06 no & $4.84 \quad \mathrm{r}$ & $4.61 \mathrm{~s}$ & $3.87 \mathrm{~m}$ & 4.21 no \\
\hline WICYT-41 & $9.03 \quad \mathrm{i}$ & $7.93 \mathrm{kl}$ & $7.84 \mathrm{f}-\mathrm{i}$ & $6.05 \mathrm{~g}$ & $5.64 \mathrm{gh}$ \\
\hline WICYT-15 & $11.95 \mathrm{gh}$ & $9.21 \mathrm{~h}$ & 6.67 no & $6.92 \mathrm{f}$ & $5.25 \mathrm{~h}-\mathrm{j}$ \\
\hline WICYT-20 & $13.77 \mathrm{de}$ & $11.33 \mathrm{de}$ & 7.59 h-j & $5.50 \quad \mathrm{i}-\mathrm{k}$ & 5.07 i-1 \\
\hline WICYT-25 & $14.96 \mathrm{c}$ & $12.23 \mathrm{c}$ & $7.08 \quad 1-n$ & $5.94 \mathrm{gh}$ & $2.03 \mathrm{v}$ \\
\hline WICYT-26 & $12.92 \mathrm{f}$ & $11.61 \mathrm{~d}$ & $7.37 \mathrm{j}-1$ & $6.75 \mathrm{f}$ & $5.89 \mathrm{fg}$ \\
\hline SATYN-2 & $15.09 \mathrm{c}$ & 8.82 hi & $6.42 \quad 0$ & $3.31 \mathrm{n}$ & $3.65 \mathrm{pq}$ \\
\hline SATYN-10 & $13.73 \mathrm{de}$ & $10.72 \mathrm{f}$ & 8.01 e-g & $6.79 \mathrm{f}$ & $5.52 \mathrm{~g}-\mathrm{i}$ \\
\hline SATYN-14 & $14.25 \mathrm{~d}$ & $11.52 \mathrm{~d}$ & $8.79 \mathrm{~d}$ & $6.71 \mathrm{f}$ & $5.14 \quad \mathrm{i}-\mathrm{k}$ \\
\hline SATYN-20 & $12.30 \mathrm{~g}$ & $8.68 \quad \mathrm{i}$ & $5.72 \mathrm{p}$ & $\begin{array}{ll}4.79 & 1 \\
\end{array}$ & $4.67 \quad 1-\mathrm{n}$ \\
\hline BARI GOM 25 & $13.62 \mathrm{e}$ & 8.93 hi & $5.20 \mathrm{qr}$ & $3.11 \mathrm{n}$ & $2.97 \mathrm{st}$ \\
\hline BARI GOM 26 & $8.94 \quad \mathrm{ij}$ & 6.83 no & $5.13 \mathrm{qr}$ & $4.88 \quad 1$ & 3.86 op \\
\hline BARI GOM 27 & $11.48 \mathrm{~h}$ & $8.56 \quad \mathrm{ij}$ & $7.72 \mathrm{~g}-\mathrm{j}$ & $7.69 \mathrm{e}$ & $7.34 \mathrm{c}$ \\
\hline BARI GOM 28 & $15.48 \mathrm{bc}$ & $12.63 \mathrm{c}$ & $9.87 \mathrm{c}$ & $8.85 \quad \mathrm{c}$ & $8.31 \mathrm{~b}$ \\
\hline BARI GOM 29 & $12.31 \mathrm{~g}$ & $9.76 \mathrm{~g}$ & $5.49 \mathrm{pq}$ & $3.08 \mathrm{n}$ & $2.30 \mathrm{uv}$ \\
\hline $\operatorname{LSD}_{(0.05)}$ & 0.61 & 0.52 & 0.41 & 0.31 & 0.46 \\
\hline CV (\%) & 4.53 & 4.86 & 4.72 & 4.24 & 7.89 \\
\hline
\end{tabular}

Values having same letter(s) do not differed significantly by least significant difference (LSD) at $5 \%$ level 
Table 6. Effect of salinity level on root dry weight of different wheat genotypes at different salt concentrations

\begin{tabular}{|c|c|c|c|c|c|c|}
\hline \multirow{3}{*}{$\begin{array}{l}\text { Genotypes } \\
\text { SATYN-22 }\end{array}$} & \multicolumn{6}{|c|}{ Root dry weight (mg) at different salt concentrations } \\
\hline & $0 \mathrm{dS} \mathrm{m}^{-1}$ & $5 \mathrm{dS} \mathrm{m}^{-1}$ & $10 \mathrm{dS} \mathrm{m}^{-1}$ & $15 \mathrm{dS} \mathrm{m}^{-1}$ & \multicolumn{2}{|c|}{$20 \mathrm{dS} \mathrm{m}^{-1}$} \\
\hline & 6.39 & $6.44 \mathrm{~g}$ & 1.61 & 2.80 & 0.00 & $\mathrm{w}$ \\
\hline SATYN-15 & 4.30 & $5.62 \mathrm{j}$ & 3.49 & $4.61 \mathrm{~g}$ & 5.63 & $\mathrm{f}$ \\
\hline SATYN-21 & 4.81 & $4.95 \mathrm{~lm}$ & 4.69 & $2.36 \mathrm{p}$ & 1.83 & tu \\
\hline SAYYN-17 & 5.16 & $6.75 \mathrm{f}$ & 4.88 & 4.17 hi & 2.77 & $\mathrm{n}$ \\
\hline SATYN-23 & 5.18 & $5.05 \quad \mathrm{k}-\mathrm{m}$ & 4.21 & $4.37 \mathrm{~h}$ & $2.55 \quad \mathrm{c}$ & op \\
\hline ESWYT-5 & $16.16 \mathrm{a}$ & $13.65 \mathrm{a}$ & $11.71 \mathrm{a}$ & $10.45 \mathrm{a}$ & 8.59 & $\mathrm{a}$ \\
\hline SATYN-24 & $4.81 \quad \mathrm{pq}$ & $5.67 \mathrm{j}$ & $5.35 \mathrm{gh}$ & $4.02 \mathrm{ij}$ & 4.13 & $\mathrm{i}$ \\
\hline ESWYT-6 & $15.37 \mathrm{~b}$ & $13.70 \mathrm{a}$ & $11.60 \quad \mathrm{a}$ & $10.09 \mathrm{~b}$ & 8.48 & $\mathrm{a}$ \\
\hline SATYN-3 & $6.05 \quad 1 \mathrm{~m}$ & $4.43 \quad \mathrm{o}$ & $3.99 \quad 1$ & $4.11 \mathrm{ij}$ & $2.37 \mathrm{p}$ & $\mathrm{pq}$ \\
\hline SATYN-27 & 5.69 & $5.77 \quad \mathrm{ij}$ & 2.16 & $3.96 \quad \mathrm{i}-\mathrm{k}$ & 2.11 & rs \\
\hline SATYN-12 & 3.83 & $3.44 \mathrm{pq}$ & 3.10 & 3.57 & $5.88 \mathrm{~d}$ & de \\
\hline SATYN-6 & 4.67 & $3.74 \mathrm{p}$ & 4.86 & 3.38 & 3.72 & $\mathrm{j}$ \\
\hline SATYN-19 & 2.64 & $1.58 \mathrm{~s}$ & 1.55 & 1.16 & 1.15 & $\mathrm{v}$ \\
\hline SATYN-16 & 5.31 no & $5.29 \mathrm{k}$ & 4.30 & 3.49 & $3.36 \quad 1$ & $\operatorname{lm}$ \\
\hline SATYN-25 & 4.67 & $5.64 \mathrm{j}$ & 5.37 & 3.94 & 3.52 & $\mathrm{kl}$ \\
\hline WICYT-7 & 3.53 & $2.02 \mathrm{r}$ & 5.84 & $2.07 \quad \mathrm{q}$ & 0.00 & $\mathrm{w}$ \\
\hline WICYT-9 & 7.45 & $4.19 \quad \mathrm{o}$ & 3.59 & 5.14 & 6.05 & $\mathrm{~cd}$ \\
\hline WICYT-28 & 8.79 & 4.49 no & 5.97 & 5.71 & 6.07 & $\mathrm{c}$ \\
\hline WICYT-35 & 7.67 & $5.25 \mathrm{kl}$ & $4.41 \quad \mathrm{k}$ & 6.95 & 4.09 & $\mathrm{i}$ \\
\hline WICYT-41 & $11.07 \mathrm{e}$ & $8.82 \quad \mathrm{~b}$ & $6.79 \mathrm{~d}$ & 7.95 & 6.27 & $\mathrm{~b}$ \\
\hline WICYT-15 & 8.88 & $6.87 \mathrm{~d}-\mathrm{f}$ & 5.16 & 5.63 & 5.29 & $\mathrm{~g}$ \\
\hline WICYT-20 & $7.78 \quad \mathrm{i}$ & $4.75 \mathrm{mn}$ & 5.07 & 4.35 & 1.99 & st \\
\hline WICYT-25 & $11.85 \mathrm{~d}$ & 6.85 ef & 3.32 & 2.34 & 1.99 & st \\
\hline WICYT-26 & $13.05 \mathrm{c}$ & $7.16 \mathrm{~cd}$ & 7.33 & 3.77 & 3.19 & $\mathrm{~m}$ \\
\hline SATYN-2 & 9.68 & $6.10 \mathrm{~h}$ & 6.35 & 3.62 & $3.64 \mathrm{j}$ & $\mathrm{jk}$ \\
\hline SATYN-10 & 6.85 & $7.28 \quad \mathrm{c}$ & 4.74 & 5.24 & $2.65 \mathrm{r}$ & no \\
\hline SATYN-14 & 8.31 & $7.09 \quad \mathrm{c}-\mathrm{e}$ & $3.03 \quad 0$ & $3.02 \quad 0$ & 2.51 & op \\
\hline SATYN-20 & 5.21 & $3.52 \mathrm{pq}$ & $2.11 \mathrm{p}$ & 1.82 & 1.20 & $\mathrm{v}$ \\
\hline BARI GOM 25 & 5.09 & $6.06 \mathrm{hi}$ & $4.20 \quad \mathrm{kl}$ & 3.95 & 2.28 & qr \\
\hline BARI GOM 26 & 7.67 & $6.59 \mathrm{fg}$ & $6.57 \mathrm{de}$ & 3.52 & 4.88 & $\mathrm{~h}$ \\
\hline BARI GOM 27 & 6.80 & $6.58 \mathrm{fg}$ & $3.59 \mathrm{~m}$ & 1.32 & 1.77 & $\mathrm{u}$ \\
\hline BARI GOM 28 & $15.08 \mathrm{~b}$ & $12.15 \mathrm{~b}$ & $10.67 \mathrm{~b}$ & $10.30 \mathrm{a}$ & 8.41 & $\mathrm{a}$ \\
\hline BARI GOM 29 & $7.37 \quad \mathrm{j}$ & $3.43 \quad \mathrm{q}$ & $1.92 \mathrm{p}$ & 1.46 & 1.17 & $\mathrm{v}$ \\
\hline $\operatorname{LSD}_{(0.05)}$ & 0.39 & 0.30 & 0.28 & 0.22 & 0.19 & \\
\hline CV (\%) & 4.2 & 4.35 & 4.64 & 4.17 & 4.31 & \\
\hline
\end{tabular}

Values having same letter(s) do not differed significantly by least significant difference (LSD) at $5 \%$ level

\subsection{Root Dry Weight}

Root dry weight of wheat genotypes severely affected by different salt concentrations (Table 6) with some exceptions of ESWYT-5, ESWYT-6 and BARI GOM 28 wheat genotypes. Root dry weight ranged from 16.16, 13.65, 11.71 and $10.45 \mathrm{mg}$ in ESWYT-5 to 2.64, 1.58, 1.55 and $1.16 \mathrm{mg}$ in SATYN-19 wheat genotypes at $0,5,10$ and $15 \mathrm{dSm}^{-1}$ salinity levels, respectively were found. At $20 \mathrm{dSm}^{-1}$ salt concentrations ESWYT-5, ESWYT-6 and BARI GOM 28 produced the maximum root dry weight $(8.59,8.49$ and 8.41 $\mathrm{mg}$, respectively) and $0.00 \mathrm{mg}$ root dry weight was found for both SATYN-22 and SATYN-12 wheat genotypes. In criteria for screening salt tolerance wheat genotype
ESWYT-5, ESWYT-6 and BARI GOM 28 wheat genotypes exhibited better tolerance against salt affected conditions irrespective of root dry weight. Dry weight is the consequence of plant physiological and biological activity. Under salt stress condition marked reduction was observed of this parameter Akbarimoghaddam et al. [3], Bhatti et al. [11] and Rumena [36]. It has been found that under stress condition photosynthetic rate is reduced markedly, spent huge energy in salt removal mechanism, reduced transportation of compatible nutrient, arrested cell division and enlargement, decreased shoot length, leaf number and ultimately reduction of plant growth and accumulation of dry matter is occurred Meiri and Poljakoff-Mayber [28], Long and Baker [27] and Seeman and Sharkey [39]. Cherian and 
Reddy [13] found that salt level $7.50 \mathrm{dSm}^{-1}$ quit detrimental for seedling, which results about $60 \%$ reduction of dry weight in Suaeda nudiflora. Decreasing of plant dry matter indicates the increasing of salinity level Sharma [41]. In this present piece of work, dry weight of seedling also adversely affected by salinity condition. The negative impact was varied among wheat genotypes which indicate different sensitivity of wheat genotypes to salt stress. Singh et al. [44]) and Moud and Maghsoudi [30] also found varied sensitivity of wheat genotypes on the basis of seedling growth in their research. Karim et al. [21] emphasizes that, seedling growth is one of the most important character for screening of salt tolerance at the early growth stage.

Table 7. Effect of salinity level on relative water content of different wheat genotypes at different salt concentrations

\begin{tabular}{|c|c|c|c|c|c|}
\hline \multirow{3}{*}{$\begin{array}{l}\text { Genotypes } \\
\text { SATYN-22 }\end{array}$} & \multicolumn{5}{|c|}{ Relative water content (\%) at different salt concentrations } \\
\hline & $0 \mathrm{dS} \mathrm{m}^{-1}$ & $5 \mathrm{dS} \mathrm{m}^{-1}$ & $10 \mathrm{dS} \mathrm{m}^{-1}$ & $15 \mathrm{dS} \mathrm{m}^{-1}$ & $20 \mathrm{dS} \mathrm{m}^{-1}$ \\
\hline & $85.30 \quad c-f$ & $87.86 \quad a b$ & $85.29 \mathrm{~cd}$ & $65.48 \mathrm{~h}$ & 0.00 \\
\hline SATYN-15 & $59.51 \quad 1$ & $54.46 \mathrm{~m}$ & 73.13 h-k & $53.5 \quad 1$ & $57.29 \mathrm{~lm}$ \\
\hline SATYN-21 & 83.42 ef & $75.40 \mathrm{gh}$ & $79.63 \quad \mathrm{e}-\mathrm{g}$ & $69.02 \mathrm{fg}$ & $69.17 \mathrm{fg}$ \\
\hline SAYYN-17 & $66.87 \mathrm{k}$ & $78.61 \quad \mathrm{e}-\mathrm{g}$ & $72.17 \quad \mathrm{i}-1$ & $78.13 \quad \mathrm{c}-\mathrm{e}$ & $79.33 \mathrm{bc}$ \\
\hline SATYN-23 & 84.89 d-f & $75.13 \mathrm{gh}$ & 69.66 k-n & $53.90 \quad 1$ & $64.11 \mathrm{~h}-\mathrm{j}$ \\
\hline ESWYT-5 & $89.80 \quad \mathrm{a}$ & $90.60 \quad \mathrm{a}$ & $92.54 \quad \mathrm{a}$ & $83.48 \quad \mathrm{a}$ & 87.39 a \\
\hline SATYN-24 & 83.96 ef & 80.00 d-f & $71.16 \quad j-m$ & $76.52 \mathrm{de}$ & $62.22 \mathrm{i}-\mathrm{k}$ \\
\hline ESWYT-6 & $89.55 \quad \mathrm{ab}$ & $90.20 \quad \mathrm{a}$ & $92.17 \quad \mathrm{ab}$ & $82.21 \quad \mathrm{ab}$ & $87.21 \quad \mathrm{a}$ \\
\hline SATYN-3 & 72.14 ij & $70.46 \quad \mathrm{i}$ & $77.01 \quad \mathrm{f}-\mathrm{h}$ & $54.79 \quad \mathrm{kl}$ & $53.92 \mathrm{~m}$ \\
\hline SATYN-27 & $88.39 \quad$ a-d & $76.87 \quad \mathrm{fg}$ & $55.09 \mathrm{r}$ & $61.12 \quad \mathrm{i}$ & $60.75 \mathrm{j}-1$ \\
\hline SATYN-12 & $74.81 \mathrm{hj}$ & 81.69 de & $73.36 \quad \mathrm{~h}-\mathrm{k}$ & 67.69 f-h & $68.92 \mathrm{fg}$ \\
\hline SATYN-6 & $78.91 \mathrm{gh}$ & $82.06 \quad \mathrm{c}-\mathrm{e}$ & $75.69 \quad \mathrm{~g}-\mathrm{i}$ & $71.04 \mathrm{f}$ & 70.75 ef \\
\hline SATYN-19 & $78.47 \mathrm{gh}$ & $76.89 \mathrm{fg}$ & $74.18 \quad h-j$ & 77.16 de & $80.54 \quad \mathrm{~b}$ \\
\hline SATYN-16 & $76.22 \mathrm{hi}$ & $82.42 \mathrm{~cd}$ & 80.03 ef & $58.72 \quad \mathrm{ij}$ & $65.53 \mathrm{~g}-\mathrm{i}$ \\
\hline SATYN-25 & $87.18 \quad$ a-e & $87.23 \quad \mathrm{ab}$ & $85.88 \quad \mathrm{~cd}$ & $81.41 \quad \mathrm{a}-\mathrm{c}$ & $76.12 \mathrm{~cd}$ \\
\hline WICYT-7 & $82.41 \quad \mathrm{fg}$ & $65.16 \quad \mathrm{j}$ & $40.03 \quad \mathrm{u}$ & $60.08 \quad \mathrm{ij}$ & $0.00 \mathrm{~s}$ \\
\hline WICYT-9 & 86.59 a-f & $61.48 \mathrm{k}$ & $63.57 \quad$ op & $61.56 \quad \mathrm{i}$ & 61.92 i-k \\
\hline WICYT-28 & 89.08 a-c & $58.53 \mathrm{kl}$ & $38.21 \quad \mathrm{u}$ & $29.28 \quad$ o & $43.62 \mathrm{p}$ \\
\hline WICYT-35 & 85.42 b-f & $39.88 \quad$ o & $65.82 \quad n-p$ & $71.19 \mathrm{f}$ & $27.86 \mathrm{r}$ \\
\hline WICYT-41 & $85.31 \quad \mathrm{c}-\mathrm{f}$ & $24.96 \mathrm{p}$ & $61.70 \mathrm{pq}$ & $58.10 \quad \mathrm{i}-\mathrm{k}$ & $44.80 \quad$ op \\
\hline WICYT-15 & $71.91 \mathrm{j}$ & $60.01 \mathrm{k}$ & $46.53 \mathrm{st}$ & $74.96 \mathrm{e}$ & $66.38 \mathrm{gh}$ \\
\hline WICYT-20 & $72.23 \quad \mathrm{ij}$ & $55.08 \quad 1 \mathrm{~m}$ & $87.99 \quad$ bc & $67.09 \mathrm{gh}$ & 78.56 bc \\
\hline WICYT-25 & $78.73 \mathrm{gh}$ & $51.63 \mathrm{~m}$ & $67.63 \mathrm{~m}-\mathrm{o}$ & $56.57 \quad \mathrm{j}-1$ & $59.66 \mathrm{kl}$ \\
\hline WICYT-26 & $78.61 \mathrm{gh}$ & $58.61 \quad \mathrm{kl}$ & $68.71 \quad 1-n$ & $35.85 \mathrm{n}$ & 46.79 n-p \\
\hline SATYN-2 & $72.31 \quad \mathrm{ij}$ & $69.32 \mathrm{i}$ & $62.21 \mathrm{pq}$ & $37.06 \mathrm{n}$ & 47.46 no \\
\hline SATYN-10 & 86.04 a-f & 85.42 bc & $50.09 \quad \mathrm{~s}$ & $54.96 \mathrm{kl}$ & $35.51 \mathrm{q}$ \\
\hline SATYN-14 & $86.34 \quad \mathrm{a}-\mathrm{f}$ & $71.05 \quad \mathrm{i}$ & $58.75 \mathrm{qr}$ & $30.01 \quad$ o & $49.09 \mathrm{n}$ \\
\hline SATYN-20 & $42.44 \quad \mathrm{~m}$ & $44.18 \mathrm{n}$ & $44.81 \quad \mathrm{t}$ & $46.99 \mathrm{~m}$ & $35.45 \mathrm{q}$ \\
\hline BARI GOM 25 & $78.04 \mathrm{~h}$ & $78.53 \quad$ e-g & $72.39 \quad \mathrm{i}-1$ & 67.81 f-h & $73.81 \mathrm{de}$ \\
\hline BARI GOM 26 & $78.78 \mathrm{gh}$ & 85.39 bc & $82.93 \mathrm{de}$ & $81.57 \quad \mathrm{ac}$ & $72.94 \mathrm{de}$ \\
\hline BARI GOM 27 & $76.32 \mathrm{hi}$ & $72.56 \quad \mathrm{hi}$ & $71.56 \quad \mathrm{i}-\mathrm{m}$ & $78.91 \quad b-d$ & $74.59 \mathrm{~d}$ \\
\hline BARI GOM 28 & 89.15 a-c & $89.89 \quad$ a & $90.91 \quad \mathrm{ab}$ & $81.96 \quad \mathrm{ab}$ & 86.64 a \\
\hline BARI GOM 29 & $72.94 \quad \mathrm{ij}$ & $54.58 \quad \mathrm{~m}$ & $59.23 \mathrm{qr}$ & $67.77 \mathrm{f}-\mathrm{h}$ & 66.37 \\
\hline $\operatorname{LSD}_{(0.05)}$ & 4.18 & 3.64 & 4.24 & 3.51 & 3.69 \\
\hline CV (\%) & 4.22 & 4.14 & 4.88 & 4.42 & 5.08 \\
\hline
\end{tabular}

Values having same letter(s) do not differed significantly by least significant difference (LSD) at $5 \%$ level 


\subsection{Relative Water Content}

Relative water content (RWC) could be the perfect most indicator of plant hydrologic condition as it denotes the physiological consequences of cellular water deficit. Water potential that possess the energy status of plant water which is effective for the transportation of water in the soil-plant-atmosphere chain. A wide range of statistical difference was observed for relative water content of wheat genotypes under different salt concentrations (Table 7). The relative water content ranged from $89.80,90.60,92.54$ and $83.48 \%$ in ESWYT-5 to $42.44 \%$ in SATYN-20; $24.96 \%$ in WICYT-41; 38.21 and $29.28 \%$ in WICYT-28 were recorded at $0,5,10$ and $15 \mathrm{dSm}^{-1}$, respectively. ESWYT-6 and BARI GOM 28 showed similar trend with ESWYT-5 in most of the salinity levels. At maximum salinity level $\left(20 \mathrm{dSm}^{-1}\right)$ relative water content ranged from $87.39 \%$ in ESWYT-5 to $0 \%$ in
SATYN-22 and WICYT-7, respectively were found. ESWYT-5, ESWYT-6 and BARI GOM 28 wheat genotypes exhibited much better performance against different salt concentrations for relative water content. Under salt stress condition tolerance plant can grow vigorously, minimize the salt uptake and maximize potential salt load per unit area by their compartmentalization technique and provide better water use efficiency thus plant growth not hampered Flower et al. [18]. Salt tolerance cultivar may be defined as the plant which has the capacity to grow under low water potential. In this point of view, high relative water content is one of tolerance technique to stress condition Sinclair and Ludlow [43]. Similar finding was reported by Sairam et al. [37] who reported that under salt stress condition, relative water content higher in salt tolerant cultivar than the sensitive one.

Table 8. Effect of salinity level on water saturation deficit of different wheat genotypes at different salt concentrations

\begin{tabular}{|c|c|c|c|c|c|c|c|c|}
\hline \multirow{3}{*}{$\begin{array}{c}\text { Treatment } \\
\text { SATYN-22 }\end{array}$} & \multicolumn{8}{|c|}{ Water saturation deficit at different salt concentrations } \\
\hline & $0 \mathrm{dS} \mathrm{m}^{-1}$ & \multicolumn{2}{|c|}{$5 \mathrm{dS} \mathrm{m}^{-1}$} & $10 \mathrm{dS} \mathrm{m}^{-1}$ & \multicolumn{2}{|c|}{$15 \mathrm{dS} \mathrm{m}^{-1}$} & \multicolumn{2}{|c|}{$20 \mathrm{dS} \mathrm{m}^{-1}$} \\
\hline & $14.70 \mathrm{kl}$ & 12.14 & q & $14.71 \mathrm{r}$ & 34.52 & $\mathrm{k}$ & 0.00 & $\mathrm{~s}$ \\
\hline SATYN-15 & $40.49 \quad \mathrm{~b}$ & 45.54 & $\mathrm{e}$ & $26.87 \operatorname{lm}$ & 57.30 & $\mathrm{c}$ & 20.67 & $\mathrm{q}$ \\
\hline SATYN-21 & 16.58 & $24.60 \quad 1$ & $\operatorname{lm}$ & $20.37 \mathrm{p}$ & 30.98 & $\operatorname{lm}$ & 30.83 & $\mathrm{n}$ \\
\hline SAYYN-17 & $10.20 \quad \mathrm{q}$ & 21.39 & $\mathrm{n}$ & $27.83 \quad \mathrm{kl}$ & 21.87 & o & 42.71 & $\mathrm{~g}$ \\
\hline SATYN-23 & $15.11 \quad \mathrm{jk}$ & 24.87 & 1 & $30.34 \quad \mathrm{ij}$ & 46.10 & $\mathrm{e}$ & 35.89 & $\mathrm{kl}$ \\
\hline ESWYT-5 & $10.20 \mathrm{q}$ & 9.40 & $\mathrm{r}$ & $7.46 \quad \mathrm{t}$ & 16.52 & $q$ & 12.61 & $\mathrm{r}$ \\
\hline SATYN-24 & $16.04 \quad \mathrm{ij}$ & 20.00 & $\mathrm{n}$ & $28.84 \quad \mathrm{jk}$ & 23.48 & no & 37.78 & $\mathrm{i}-\mathrm{k}$ \\
\hline ESWYT-6 & $10.45 \mathrm{pq}$ & 9.80 & $\mathrm{r}$ & $7.83 \quad \mathrm{t}$ & 17.79 & $q$ & 12.79 & $r$ \\
\hline SATYN-3 & $27.86 \quad \mathrm{~d}$ & 29.54 & $\mathrm{ij}$ & $22.99 \quad$ o & 45.21 & ef & 46.06 & $\mathrm{f}$ \\
\hline SATYN-27 & 11.61 ор & 23.13 & $\mathrm{~m}$ & $44.91 \mathrm{~d}$ & 53.01 & $\mathrm{~d}$ & 39.25 & hi \\
\hline SATYN-12 & $10.45 \mathrm{pq}$ & 18.31 & o & $26.64 \quad 1 \mathrm{~m}$ & 32.31 & $\mathrm{kl}$ & 31.08 & $\mathrm{n}$ \\
\hline SATYN-6 & $21.09 \mathrm{~g}$ & 17.94 & o & 24.31 no & 28.96 & $\mathrm{~m}$ & 29.25 & $\mathrm{n}$ \\
\hline SATYN-19 & $21.53 \mathrm{~g}$ & $23.11 \mathrm{n}$ & $\mathrm{m}$ & $25.82 \mathrm{mn}$ & 22.84 & no & 19.46 & $q$ \\
\hline SATYN-16 & $23.78 \mathrm{f}$ & 17.58 & o & $19.97 \mathrm{p}$ & 41.28 & g-i & 34.47 & $1 \mathrm{~m}$ \\
\hline SATYN-25 & 12.82 no & 12.77 & $q$ & $14.12 \mathrm{r}$ & 18.59 & $\mathrm{pq}$ & 23.88 & $\mathrm{p}$ \\
\hline WICYT-7 & $17.59 \mathrm{~h}$ & 34.84 & $\mathrm{~h}$ & $59.97 \quad \mathrm{a}$ & 39.92 & $h-j$ & 0.00 & $\mathrm{~s}$ \\
\hline WICYT-9 & $13.41 \mathrm{mn}$ & 38.52 & $\mathrm{~g}$ & $36.43 \mathrm{f}$ & 38.44 & $\mathrm{j}$ & 38.08 & $\mathrm{ij}$ \\
\hline WICYT-28 & $10.92 \mathrm{pq}$ & 56.32 & $\mathrm{c}$ & $61.72 \quad \mathrm{a}$ & 70.72 & $\mathrm{a}$ & 56.38 & $\mathrm{c}$ \\
\hline WICYT-35 & $14.58 \quad \mathrm{k}-\mathrm{m}$ & 60.52 & $\mathrm{~b}$ & $55.19 \mathrm{~b}$ & 28.81 & $\mathrm{~m}$ & 72.14 & $\mathrm{a}$ \\
\hline WICYT-41 & $14.69 \mathrm{kl}$ & 75.04 & $\mathrm{a}$ & $38.30 \quad \mathrm{f}$ & 41.90 & gh & 55.20 & $\mathrm{~cd}$ \\
\hline WICYT-15 & $28.09 \quad \mathrm{~d}$ & $39.99 \quad 1$ & $\mathrm{fg}$ & $53.47 \quad \mathrm{~b}$ & 25.04 & $\mathrm{n}$ & 33.62 & $\mathrm{~m}$ \\
\hline WICYT-20 & 33.13 & 44.92 & $\mathrm{e}$ & $12.01 \mathrm{~s}$ & 32.91 & $\mathrm{kl}$ & 21.44 & $q$ \\
\hline WICYT-25 & 25.19 & 55.82 & $\mathrm{c}$ & $32.37 \mathrm{gh}$ & 43.43 & $\mathrm{fg}$ & 40.34 & $\mathrm{~h}$ \\
\hline WICYT-26 & $21.39 \mathrm{~g}$ & 41.40 & $\mathrm{f}$ & 31.29 hi & 64.15 & $\mathrm{~b}$ & 53.21 & de \\
\hline SATYN-2 & $27.69 \mathrm{~d}$ & 30.68 & $\mathrm{i}$ & $37.79 \mathrm{f}$ & 62.94 & $\mathrm{~b}$ & 52.54 & $\mathrm{e}$ \\
\hline SATYN-10 & $13.96 \quad$ k-n & 14.58 & $\mathrm{p}$ & $49.91 \mathrm{c}$ & 45.04 & ef & 64.49 & $\mathrm{~b}$ \\
\hline SATYN-14 & $13.66 \quad 1-n$ & $28.95 \mathrm{j}$ & $\mathrm{jk}$ & $41.25 \mathrm{e}$ & 69.99 & $\mathrm{a}$ & 51.91 & $\mathrm{e}$ \\
\hline SATYN-20 & $57.56 \quad \mathrm{a}$ & 48.37 & $\mathrm{~d}$ & $34.19 \mathrm{~g}$ & 38.88 & ij & 36.15 & $\mathrm{j}-1$ \\
\hline BARI GOM 25 & $21.96 \mathrm{~g}$ & 21.47 & $\mathrm{n}$ & $27.61 \quad \mathrm{k}-\mathrm{m}$ & 32.19 & $\mathrm{kl}$ & 26.19 & o \\
\hline BARI GOM 26 & $21.22 \mathrm{~g}$ & 9.804 & $\mathrm{r}$ & $17.07 \quad \mathrm{q}$ & 18.43 & $\mathrm{q}$ & 27.06 & o \\
\hline BARI GOM 27 & $23.68 \mathrm{f}$ & 27.43 & $\mathrm{k}$ & $28.44 \quad \mathrm{j}-1$ & 21.09 & op & 25.41 & op \\
\hline BARI GOM 28 & $10.85 \mathrm{pq}$ & 10.11 & $\mathrm{r}$ & 9.09 & 18.04 & $\mathrm{q}$ & 13.36 & $\mathrm{r}$ \\
\hline BARI GOM 29 & $27.06 \mathrm{~d}$ & 45.42 & $\mathrm{e}$ & $40.77 \quad \mathrm{e}$ & 32.23 & $\mathrm{kl}$ & 33.363 & $\mathrm{~m}$ \\
\hline $\operatorname{LSD}_{(0.05)}$ & 1.26 & 1.61 & & 1.95 & 2.53 & & 2.10 & \\
\hline CV (\%) & 4.83 & 4.26 & & 5.1 & 5.49 & & 5.11 & \\
\hline
\end{tabular}

Values having same letter(s) do not differed significantly by least significant difference (LSD) at 5\% level 


\subsection{Water Saturation Deficit}

The amount of water vapor needs to be increased in the air to attain a saturation point without disturbing the environmental condition (temperature and pressure) is called water saturation deficit. It is opposite to relative water content. Salinity level had highly significant influence on water saturation deficit among different wheat genotypes (Table 8). The result revealed that, water saturation deficit ranged from 57.56 in SATYN-20, 75.04 in WICYT-41, 61.72 and 70.72 in WICYT-28 to $10.20,9.40,7.46$ and 16.52 in ESWYT-5 at 0, 5, 10 and $15 \mathrm{dSm}^{-1}$, respectively were observed. At $20 \mathrm{dSm}^{-1}$ the maximum water saturation deficit
72.14 was observed in WICYT-35. SATYN-22 and WICYT-7 showed very much sensitivity to higher salt concentration $\left(20 \mathrm{dSm}^{-1}\right)$ and those were not survived. Therefore, ESWYT-5, ESWYT-6 and BARI GOM 28 wheat genotypes exerted better tolerance against salinity condition in case of water saturation deficit. Due to lack of compartmentalization technique, the salinity sensitive cultivars failed to uptake enough water necessary for running the physiological process smoothly under salt stress condition, thus there was a huge water deficit occurred in sensitive cultivars than the tolerance cultivars. Similar result was reported by Baque et al. [9].

Table 9. Effect of salinity level on water retention capacity of different wheat genotypes at different salt concentrations

\begin{tabular}{|c|c|c|c|c|c|}
\hline \multirow{3}{*}{$\begin{array}{l}\text { Genotypes } \\
\text { SATYN-22 }\end{array}$} & \multicolumn{5}{|c|}{ Water retention capacity at different salt concentrations } \\
\hline & $0 \mathrm{dS} \mathrm{m}^{-1}$ & $5 \mathrm{dS} \mathrm{m}^{-1}$ & $10 \mathrm{dS} \mathrm{m}^{-1}$ & $15 \mathrm{dS} \mathrm{m}^{-1}$ & $20 \mathrm{dS} \mathrm{m}^{-1}$ \\
\hline & 8.62 & $8.772 \mathrm{q}$ & $12.35 \mathrm{bc}$ & 8.53 i-k & $0.00 \mathrm{t}$ \\
\hline SATYN-15 & $12.19 \mathrm{ij}$ & $12.45 \mathrm{fg}$ & $11.28 \mathrm{de}$ & $9.69 \mathrm{f}$ & $6.85 \mathrm{pq}$ \\
\hline SATYN-21 & 9.70 & $13.13 \mathrm{de}$ & 10.73 e-g & $7.94 \mathrm{kl}$ & $10.24 \mathrm{e}-\mathrm{g}$ \\
\hline SAYYN-17 & $12.21 \mathrm{ij}$ & $11.68 \mathrm{hi}$ & $11.84 \mathrm{~cd}$ & $8.99 \mathrm{~g}-\mathrm{i}$ & 7.81 no \\
\hline SATYN-23 & 9.178 ор & $11.25 \mathrm{ij}$ & $9.634 \mathrm{jk}$ & $9.05 \mathrm{~g}-\mathrm{i}$ & $7.87 \quad \mathrm{~m}-\mathrm{o}$ \\
\hline ESWYT-5 & $16.40 \mathrm{a}$ & $24.60 \mathrm{a}$ & $13.93 \mathrm{a}$ & $12.06 \mathrm{a}$ & $19.34 \quad \mathrm{a}$ \\
\hline SATYN-24 & $10.59 \mathrm{~m}$ & $12.54 \mathrm{e}-\mathrm{g}$ & $10.61 \mathrm{f}-\mathrm{h}$ & $8.02 \mathrm{kl}$ & $8.43 \mathrm{k}-\mathrm{m}$ \\
\hline ESWYT-6 & $15.95 \mathrm{a}$ & 24.14 a & $12.61 \mathrm{~b}$ & $11.91 \mathrm{a}$ & $10.18 \quad \mathrm{e}-\mathrm{g}$ \\
\hline SATYN-3 & $10.88 \mathrm{~lm}$ & $13.26 \mathrm{~d}$ & $8.95 \mathrm{~lm}$ & $10.34 \mathrm{e}$ & 9.06 \\
\hline SATYN-27 & $10.16 \mathrm{mn}$ & $9.982 \quad 1-n$ & $9.41 \quad \mathrm{kl}$ & $9.25 \quad \mathrm{f}-\mathrm{h}$ & 6.46 \\
\hline SATYN-12 & $12.92 \mathrm{f}-\mathrm{i}$ & $9.700 \quad \mathrm{~m}-\mathrm{o}$ & $6.70 \mathrm{p}$ & $10.57 \mathrm{de}$ & $10.44 \mathrm{~d}-\mathrm{f}$ \\
\hline SATYN-6 & $10.69 \mathrm{~lm}$ & $11.20 \mathrm{ij}$ & 9.58 & $10.54 \mathrm{de}$ & $10.63 \mathrm{de}$ \\
\hline SATYN-19 & $10.54 \mathrm{~m}$ & $10.26 \mathrm{k}-\mathrm{m}$ & $10.65 \mathrm{f}-\mathrm{h}$ & $10.78 \quad \mathrm{c}-\mathrm{e}$ & 7.82 \\
\hline SATYN-16 & $11.38 \mathrm{kl}$ & $11.16 \mathrm{ij}$ & $10.37 \mathrm{~g}-\mathrm{i}$ & $10.53 \mathrm{de}$ & $10.71 \mathrm{de}$ \\
\hline SATYN-25 & $12.61 \mathrm{~g}-\mathrm{i}$ & $13.06 \mathrm{~d}-\mathrm{f}$ & $10.09 \mathrm{~h}-\mathrm{j}$ & $8.21 \quad \mathrm{j}-1$ & 9.55 \\
\hline WICYT-7 & $13.09 \mathrm{~d}-\mathrm{g}$ & $9.04 \quad \mathrm{o}-\mathrm{q}$ & $6.45 \mathrm{pq}$ & 9.71 & 0.00 \\
\hline WICYT-9 & $12.25 \mathrm{~h}-\mathrm{j}$ & $17.73 \mathrm{c}$ & $5.04 \quad \mathrm{~s}$ & 7.72 & 8.16 \\
\hline WICYT-28 & $13.55 \mathrm{c}-\mathrm{f}$ & $9.46 \quad n-p$ & $5.78 \mathrm{r}$ & 5.23 & 11.33 \\
\hline WICYT-35 & 9.444 no & $11.01 \mathrm{ij}$ & $5.80 \mathrm{r}$ & 6.81 & 5.46 \\
\hline WICYT-41 & $13.76 \mathrm{~cd}$ & $5.82 \mathrm{t}$ & 6.94 op & 5.07 & 4.79 \\
\hline WICYT-15 & $13.43 \mathrm{c}-\mathrm{f}$ & $7.022 \mathrm{r}$ & $8.26 \mathrm{n}$ & 6.83 & $7.36 \quad$ op \\
\hline WICYT-20 & $10.87 \mathrm{~lm}$ & $6.338 \mathrm{st}$ & $8.54 \mathrm{mn}$ & 9.19 & 6.36 \\
\hline WICYT-25 & $10.84 \mathrm{~lm}$ & $8.792 \mathrm{pq}$ & $5.96 \mathrm{qr}$ & $8.74 \quad h-j$ & 8.84 \\
\hline WICYT-26 & $11.80 \mathrm{jk}$ & $12.26 \mathrm{gh}$ & $6.62 \mathrm{p}$ & 6.32 & 9.03 \\
\hline SATYN-2 & $11.82 \mathrm{jk}$ & $11.22 \mathrm{ij}$ & $7.42 \quad 0$ & 5.60 & 9.69 \\
\hline SATYN-10 & $9.28 \quad$ ор & $17.18 \mathrm{c}$ & $9.66 \mathrm{jk}$ & $6.862 \mathrm{~m}$ & 8.73 \\
\hline SATYN-14 & $11.75 \mathrm{jk}$ & $6.80 \quad \mathrm{rs}$ & $6.47 \mathrm{pq}$ & $8.76 \quad h-j$ & $8.27 \quad \mathrm{k}-\mathrm{n}$ \\
\hline SATYN-20 & 9.538 no & $7.22 \quad \mathrm{r}$ & $4.92 \mathrm{~s}$ & $10.8 \quad \mathrm{c}-\mathrm{e}$ & 9.55 \\
\hline BARI GOM 25 & 12.97 e-h & $13.22 \mathrm{~d}$ & $11.94 \quad \mathrm{c}$ & $11.28 \mathrm{bc}$ & $9.68 \mathrm{gh}$ \\
\hline BARI GOM 26 & $14.15 \mathrm{bc}$ & $10.58 \quad \mathrm{j}-1$ & 10.96 ef & $9.58 \quad \mathrm{fg}$ & $10.04 \mathrm{f}-\mathrm{h}$ \\
\hline BARI GOM 27 & $12.66 \mathrm{~g}-\mathrm{i}$ & $12.11 \mathrm{gh}$ & $9.17 \quad \mathrm{kl}$ & 10.99 b-d & $10.82 \mathrm{~cd}$ \\
\hline BARI GOM 28 & $14.87 \mathrm{~b}$ & $21.23 \mathrm{~b}$ & $12.11 \mathrm{bc}$ & $11.50 \mathrm{ab}$ & $15.78 \quad \mathrm{~b}$ \\
\hline BARI GOM 29 & $13.68 \mathrm{c}-\mathrm{e}$ & $10.85 \mathrm{jk}$ & $10.03 \mathrm{ij}$ & 9.65 & $8.14 \mathrm{mn}$ \\
\hline $\operatorname{LSD}_{(0.05)}$ & 0.75 & 0.68 & 0.58 & 0.60 & 0.58 \\
\hline CV (\%) & 5.07 & 4.69 & 5.05 & 5.36 & 5.47 \\
\hline
\end{tabular}

Values having same letter(s) do not differed significantly by least significant difference (LSD) at $5 \%$ level 


\subsection{Water Retention Capacity}

The amount of water useful for crop hold by the crop plant is the water retention capacity. Different salt concentrations significantly influenced water retention capacity of wheat genotypes (Table 9). Highest water retention capacity ranged from 16.40, 24.60, 13.93, 12.06 19.34 in ESWYT-5 to 8.62 in SATYN-22, 5.82 in WICYT-41, 4.92 in SATYN-20, 5.07 in WICYT-41 and 0 in both SATYN-22 and WICYT-7 at 0 , $5,10,15$ and $20 \mathrm{dSm}^{-1}$, respectively were observed. The tolerance cultivars have the capacity to uptake water under salt stress condition than the sensitive ones and gained the maximum turgid weight, in consequence they gained the maximum water retention capacity. The result also coincides with the result of Sangakkara et al. [38].

Table 10. Effect of salinity level on coefficient of germination of different wheat genotypes at different salt concentrations

\begin{tabular}{|c|c|c|c|c|c|}
\hline \multirow{3}{*}{$\begin{array}{l}\text { Genotypes } \\
\text { SATYN-22 }\end{array}$} & \multicolumn{5}{|c|}{ Coefficient of germination at different salt concentrations } \\
\hline & $0 \mathrm{dS} \mathrm{m}^{-1}$ & $5 \mathrm{dS} \mathrm{m}^{-1}$ & \multirow{2}{*}{$\begin{array}{c}10 \mathrm{dS} \mathrm{m}^{-1} \\
13.61 \mathrm{j}\end{array}$} & $15 \mathrm{dS} \mathrm{m}^{-1}$ & $20 \mathrm{dS} \mathrm{m}^{-1}$ \\
\hline & 15.89 a-h & $14.33 \mathrm{jk}$ & & $14.73 \quad \mathrm{e}-\mathrm{i}$ & $13.81 \quad \mathrm{~h}-\mathrm{k}$ \\
\hline SATYN-15 & 15.76 a-i & $14.84 \mathrm{~g}-\mathrm{jk}$ & $14.14 \mathrm{~g}-\mathrm{j}$ & $15.01 \quad \mathrm{e}-\mathrm{i}$ & $12.97 \mathrm{k}$ \\
\hline SATYN-21 & 16.04 a-f & $15.43 \quad \mathrm{~d}-\mathrm{i}$ & $14.58 \mathrm{~d}-\mathrm{j}$ & $15.57 \quad b-e$ & $13.45 \quad \mathrm{i}-\mathrm{k}$ \\
\hline SAYYN-17 & $15.35 \mathrm{~d}-\mathrm{k}$ & $15.02 \mathrm{f}-\mathrm{j}$ & $14.06 \mathrm{~h}-\mathrm{j}$ & 14.43 g-j & $14.14 \mathrm{f}-\mathrm{i}$ \\
\hline SATYN-23 & $15.43 \mathrm{c}-\mathrm{k}$ & $15.43 \mathrm{~d}-\mathrm{i}$ & $14.13 \mathrm{~g}-\mathrm{j}$ & $14.91 \quad \mathrm{e}-\mathrm{i}$ & 13.89 g-k \\
\hline ESWYT-5 & $16.56 \mathrm{a}$ & $16.68 \quad \mathrm{a}$ & 16.48 a & $16.78 \quad \mathrm{a}$ & $17.33 \quad \mathrm{a}$ \\
\hline SATYN-24 & $15.26 \mathrm{f}-\mathrm{k}$ & $15.00 \quad \mathrm{f}-\mathrm{k}$ & $15.09 \mathrm{c}-\mathrm{g}$ & $14.76 \quad \mathrm{e}-\mathrm{i}$ & $14.27 \quad$ e-i \\
\hline ESWYT-6 & $16.53 \mathrm{a}$ & $16.63 \mathrm{ab}$ & $16.47 \mathrm{a}$ & $16.59 \mathrm{a}$ & $16.69 \mathrm{ab}$ \\
\hline SATYN-3 & 15.64 a-j & $14.89 \mathrm{~g}-\mathrm{k}$ & $14.68 \mathrm{~d}-\mathrm{i}$ & $14.17 \quad \mathrm{ij}$ & 13.88 g-k \\
\hline SATYN-27 & 15.30 e-k & $13.98 \mathrm{k}$ & $14.13 \mathrm{~g}-\mathrm{j}$ & $14.14 \quad \mathrm{ij}$ & 14.59 e-h \\
\hline SATYN-12 & 15.77 a-i & $14.67 \quad \mathrm{i}-\mathrm{k}$ & $13.71 \mathrm{ij}$ & $14.82 \mathrm{e}-\mathrm{i}$ & 14.46 e-h \\
\hline SATYN-6 & 16.20 a-e & $15.52 \quad \mathrm{c}-\mathrm{i}$ & $14.50 \quad e-j$ & $15.25 \mathrm{c}-\mathrm{g}$ & 14.92 d-f \\
\hline SATYN-19 & 16.07 a-f & $14.88 \quad \mathrm{~g}-\mathrm{k}$ & $14.39 \mathrm{f}-\mathrm{j}$ & $14.37 \mathrm{~g}-\mathrm{j}$ & 14.63 e-h \\
\hline SATYN-16 & 15.84 a-h & $14.67 \quad \mathrm{i}-\mathrm{k}$ & $14.70 \mathrm{~d}-\mathrm{h}$ & $14.16 \mathrm{ij}$ & $14.18 \quad \mathrm{f}-\mathrm{i}$ \\
\hline SATYN-25 & $16.01 \mathrm{a}-\mathrm{g}$ & $15.26 \quad e-j$ & 14.56 e-j & $14.21 \mathrm{~h}-\mathrm{j}$ & $14.06 \quad \mathrm{f}-\mathrm{j}$ \\
\hline WICYT-7 & 16.11 a-f & 16.26 a-e & $14.74 \mathrm{~d}-\mathrm{h}$ & $14.63 \mathrm{f}-\mathrm{i}$ & $14.02 \mathrm{f}-\mathrm{j}$ \\
\hline WICYT-9 & $16.26 \quad a-d$ & $15.61 \quad b-i$ & $16.24 \mathrm{ab}$ & $14.63 \mathrm{f}-\mathrm{i}$ & $16.51 \mathrm{ab}$ \\
\hline WICYT-28 & 16.06 a-f & $14.90 \quad \mathrm{~g}-\mathrm{k}$ & $16.29 \mathrm{ab}$ & $15.09 \mathrm{~d}-\mathrm{h}$ & $16.39 \mathrm{ab}$ \\
\hline WICYT-35 & 15.84 a-h & 15.79 a-g & $14.35 \mathrm{f}-\mathrm{j}$ & $13.67 \mathrm{j}$ & $16.40 \quad \mathrm{ab}$ \\
\hline WICYT-41 & $15.07 \mathrm{~h}-\mathrm{k}$ & 16.23 a-e & $15.17 \mathrm{c}-\mathrm{f}$ & 15.98 a-d & $14.81 \quad \mathrm{~d}-\mathrm{g}$ \\
\hline WICYT-15 & 16.14 a-f & 16.43 a-d & $16.22 \mathrm{ab}$ & $16.51 \mathrm{a}$ & $16.50 \quad \mathrm{ab}$ \\
\hline WICYT-20 & 16.21 a-e & 16.01 a-f & $16.26 \mathrm{ab}$ & $16.58 \mathrm{a}$ & $16.08 \quad b c$ \\
\hline WICYT-25 & 15.31 e-k & $15.00 \quad \mathrm{f}-\mathrm{k}$ & 15.95 a-c & $16.37 \mathrm{ab}$ & $16.29 \quad \mathrm{~b}$ \\
\hline WICYT-26 & $15.47 \quad b-j$ & $15.70 \quad$ a-h & 15.92 a-c & 15.99 a-d & $15.24 \quad \mathrm{c}-\mathrm{e}$ \\
\hline SATYN-2 & 16.36 a-c & 16.45 a-d & $16.46 \mathrm{a}$ & $16.22 \mathrm{ab}$ & $16.13 \mathrm{bc}$ \\
\hline SATYN-10 & 15.85 a-h & 16.00 a-f & $16.17 \mathrm{ab}$ & 16.04 a-c & $15.78 \quad b-d$ \\
\hline SATYN-14 & $15.58 \quad b-j$ & $15.40 \quad$ e-i & 15.55 a-d & $15.51 \quad b-f$ & $16.09 \mathrm{bc}$ \\
\hline SATYN-20 & $15.59 \quad b-j$ & $15.26 \quad e-j$ & $15.43 \mathrm{~b}-\mathrm{e}$ & $16.02 \mathrm{a}-\mathrm{c}$ & $14.27 \quad$ e- $\mathrm{i}$ \\
\hline BARI GOM 25 & $14.89 \quad \mathrm{i}-\mathrm{k}$ & 14.74 h-k & $13.98 \mathrm{~h}-\mathrm{j}$ & $13.68 \quad \mathrm{j}$ & $13.15 \quad \mathrm{jk}$ \\
\hline BARI GOM 26 & 15.11 g-k & 14.92 g-k & $14.28 \mathrm{f}-\mathrm{j}$ & $14.78 \quad \mathrm{e}-\mathrm{i}$ & 14.72 \\
\hline BARI GOM 27 & $14.77 \quad \mathrm{jk}$ & $14.62 \quad \mathrm{i}-\mathrm{k}$ & 14.87 d-h & $14.42 \mathrm{~g}-\mathrm{j}$ & 14.36 \\
\hline BARI GOM 28 & $16.40 \mathrm{ab}$ & $16.50 \quad a-c$ & $16.44 \mathrm{a}$ & $16.63 \mathrm{a}$ & $16.67 \quad \mathrm{ab}$ \\
\hline BARI GOM 29 & $14.52 \mathrm{k}$ & $14.84 \quad$ g-k & $14.27 \mathrm{f}-\mathrm{j}$ & $14.34 \quad h-j$ & 14.49 e-h \\
\hline $\operatorname{LSD}_{(0.05)}$ & 0.93 & 1.02 & 0.97 & 0.90 & 0.99 \\
\hline CV (\%) & 4.72 & 5.31 & 5.15 & 4.7 & 5.28 \\
\hline
\end{tabular}

Values having same letter(s) do not differed significantly by least significant difference (LSD) at $5 \%$ level 


\subsection{Coefficient of Germination}

Salinity levels significantly influenced the coefficient of germination of wheat genotypes (Table 10). Maximum coefficient of germination ranged from 16.56, 16.68, 16.48 16.78 and 17.33 in ESWYT-5 to 14.52 in BARI GOM 29, 13.98 in SATYN-27, 13.61 in SATYN-22, 13.67 in WICYT-35 and 12.97 in SATYN-15 at 0, 5, 10, 15 and 20
$\mathrm{dSm}^{-1}$, respectively were recorded. The reduction of germination rate with the increasing of salt concentration occurred for salt sensitive cultivars which caused reduction of coefficient of germination under salt stress condition. Similar results were reported by Akbarimoghaddam et al. [3], Datta et al. [17], Mujeeb-ur-Rahman et al. [31], Rahman et al. [34], Singh et al. [44] and Mirza and Mahmood [29].

Table 11. Effect of salinity level on vigor index of different wheat genotypes at different salt concentrations

\begin{tabular}{|c|c|c|c|c|c|}
\hline \multirow{3}{*}{$\begin{array}{l}\text { Genotypes } \\
\text { SATYN-22 }\end{array}$} & \multicolumn{5}{|c|}{ Vigour index at different salt concentrations } \\
\hline & $0 \mathrm{dS} \mathrm{m}^{-1}$ & $5 \mathrm{dS} \mathrm{m}^{-1}$ & $10 \mathrm{dS} \mathrm{m}^{-1}$ & $15 \mathrm{dS} \mathrm{m}^{-1}$ & $20 \mathrm{dS} \mathrm{m}^{-1}$ \\
\hline & $213.7 \quad \mathrm{ij}$ & $152.7 \mathrm{gh}$ & 39.11 rs & $60.41 \quad \mathrm{j}$ & 0.00 \\
\hline SATYN-15 & $251.0 \quad b-d$ & $170.7 \mathrm{e}$ & $73.32 \mathrm{jk}$ & $119.6 \mathrm{c}$ & $42.59 \mathrm{i}$ \\
\hline SATYN-21 & 223.2 g-i & $195.7 \mathrm{~d}$ & $141.3 \mathrm{~d}$ & $103.4 \mathrm{e}$ & $46.09 \mathrm{~h}$ \\
\hline SAYYN-17 & $191.8 \mathrm{~m}$ & $137.1 \quad \mathrm{i}$ & 81.85 i & $61.02 \mathrm{j}$ & 24.69 o \\
\hline SATYN-23 & $246.9 \quad$ c-e & $173.5 \mathrm{e}$ & $109.2 \mathrm{f}$ & 98.76 ef & $32.39 \mathrm{kl}$ \\
\hline ESWYT-5 & $270.5 \quad \mathrm{a}$ & $287.7 \quad \mathrm{a}$ & $210.5 \quad \mathrm{a}$ & $155.78 \mathrm{a}$ & $164.8 \mathrm{a}$ \\
\hline SATYN-24 & $179.7 \mathrm{n}$ & $195.0 \mathrm{~d}$ & $124.1 \mathrm{e}$ & $113.1 \mathrm{~d}$ & $82.13 \mathrm{~d}$ \\
\hline ESWYT-6 & $270.7 \quad \mathrm{a}$ & $252.6 \mathrm{~b}$ & $205.57 \mathrm{a}$ & $152.7 \quad \mathrm{a}$ & $162.89 \mathrm{a}$ \\
\hline SATYN-3 & $167.1 \quad \mathrm{o}$ & $103.8 \mathrm{k}$ & $46.18 \quad \mathrm{pq}$ & $28.62 \mathrm{pq}$ & 26.15 no \\
\hline SATYN-27 & $163.6 \quad$ o & $133.5 \mathrm{i}$ & $39.40 \quad$ rs & $33.58 \quad \mathrm{p}$ & $16.61 \mathrm{p}$ \\
\hline SATYN-12 & $107.5 \quad \mathrm{q}$ & $85.51 \quad \mathrm{~lm}$ & $68.96 \mathrm{kl}$ & 47.17 no & $33.35 \mathrm{jkl}$ \\
\hline SATYN-6 & 238.6 ef & $175.1 \quad \mathrm{e}$ & $97.68 \mathrm{gh}$ & $93.83 \mathrm{fg}$ & $28.37 \mathrm{mn}$ \\
\hline SATYN-19 & $127.1 \quad \mathrm{p}$ & $115.6 \mathrm{j}$ & $77.83 \quad \mathrm{ij}$ & $43.04 \quad 0$ & $62.47 \mathrm{f}$ \\
\hline SATYN-16 & $222.4 \quad$ hi & $196.8 \mathrm{~d}$ & $137.5 \mathrm{~d}$ & $92.55 \mathrm{gh}$ & $71.54 \mathrm{e}$ \\
\hline SATYN-25 & $202.3 \quad \mathrm{k}-\mathrm{m}$ & $207.9 \mathrm{c}$ & $99.30 \mathrm{~g}$ & 88.15 hi & 0.00 \\
\hline WICYT-7 & $207.4 \quad \mathrm{j}-1$ & $66.51 \quad$ o & $12.70 \quad \mathrm{u}$ & $55.18 \quad \mathrm{kl}$ & $14.33 \mathrm{p}$ \\
\hline WICYT-9 & 239.9 d-f & $53.43 \mathrm{p}$ & $36.08 \mathrm{~s}$ & $59.54 \quad \mathrm{jk}$ & $46.87 \mathrm{~h}$ \\
\hline WICYT-28 & $213.5 \quad \mathrm{i}-\mathrm{k}$ & $42.32 \mathrm{q}$ & $71.71 \quad \mathrm{jk}$ & $50.39 \quad 1-n$ & 27.87 no \\
\hline WICYT-35 & $216.1 \quad \mathrm{ij}$ & $23.40 \mathrm{st}$ & $47.49 \quad$ op & $86.35 \quad \mathrm{i}$ & $36.47 \mathrm{j}$ \\
\hline WICYT-41 & $234.0 \quad \mathrm{fg}$ & $91.82 \quad 1$ & $58.93 \mathrm{mn}$ & $100.9 \quad \mathrm{e}$ & $31.34 \quad \mathrm{~lm}$ \\
\hline WICYT-15 & $207.6 \quad j-1$ & 75.67 no & $74.44 \quad \mathrm{jk}$ & $52.59 \quad 1 \mathrm{~m}$ & $48.05 \mathrm{~h}$ \\
\hline WICYT-20 & $231.5 \quad \mathrm{f}-\mathrm{h}$ & $47.51 \mathrm{pq}$ & $147.9 \quad \mathrm{c}$ & 98.35 ef & $53.62 \mathrm{~g}$ \\
\hline WICYT-25 & $120.4 \mathrm{p}$ & $32.60 \mathrm{r}$ & $43.47 \quad$ p-r & $13.22 \mathrm{~s}$ & $16.00 \mathrm{p}$ \\
\hline WICYT-26 & 240.6 d-f & $147.1 \mathrm{~h}$ & $63.33 \quad 1 \mathrm{~m}$ & 45.97 no & $96.80 \mathrm{c}$ \\
\hline SATYN-2 & $248.8 \quad$ c-e & $161.1 \mathrm{fg}$ & $71.60 \quad \mathrm{jk}$ & $22.76 \mathrm{r}$ & $24.51 \quad$ o \\
\hline SATYN-10 & 216.3 ij & 169.2 ef & 53.58 no & $63.01 \mathrm{j}$ & $34.92 \quad \mathrm{jk}$ \\
\hline SATYN-14 & $197.9 \quad \mathrm{~lm}$ & $131.2 \mathrm{i}$ & $48.19 \quad$ ор & $16.64 \mathrm{~s}$ & 27.77 no \\
\hline SATYN-20 & $68.94 \mathrm{~s}$ & $16.64 \mathrm{t}$ & $7.654 \quad \mathrm{u}$ & $6.268 \mathrm{t}$ & $1.790 \quad \mathrm{q}$ \\
\hline BARI GOM 25 & $91.00 \quad \mathrm{r}$ & $81.09 \mathrm{mn}$ & $23.52 \mathrm{t}$ & $23.74 \mathrm{qr}$ & $15.60 \quad \mathrm{p}$ \\
\hline BARI GOM 26 & $234.1 \mathrm{fg}$ & $189.6 \mathrm{~d}$ & $92.54 \mathrm{~h}$ & $48.75 \mathrm{mn}$ & $52.66 \mathrm{~g}$ \\
\hline BARI GOM 27 & $258.0 \quad b c$ & $130.2 \mathrm{i}$ & 40.07 qrs & $13.74 \mathrm{~s}$ & $16.49 \mathrm{p}$ \\
\hline BARI GOM 28 & $262.0 \quad \mathrm{ab}$ & $244.6 \mathrm{~b}$ & $163.3 \quad \mathrm{~b}$ & $134.6 \quad \mathrm{~b}$ & $105.3 \quad \mathrm{~b}$ \\
\hline BARI GOM 29 & $95.81 \quad \mathrm{r}$ & 32.16 rs & $7.186 \quad \mathrm{u}$ & $5.544 \mathrm{t}$ & $1.688 \quad \mathrm{q}$ \\
\hline $\operatorname{LSD}_{(0.05)}$ & 11.38 & 9.17 & 6.40 & 5.1 & 3.34 \\
\hline CV (\%) & 4.51 & 5.6 & 6.66 & 6.58 & 6.88 \\
\hline
\end{tabular}

Values having same letter(s) do not differed significantly by least significant difference (LSD) at $5 \%$ level 


\subsection{Vigour Index}

Salinity levels significantly affected vigour index among different wheat genotypes (table 11). The magnitude of reduction of vigour index was slow in case of ESWYT-5 followed by ESWYT-6 and BARI GOM 28ie.they hold a consistently decreasing trend but most of wheat genotypes exerted rapid reduction of vigour index with the increasing of salinity level. ESWYT-5 scored the maximum vigour index $\left(287.70,210.5,155.78\right.$ and 164.80 at $5,10,15$ and $20 \mathrm{dSm}^{-1}$, respectively) but at control ESWYT-6 scored the maximum vigour index (270.70) which showed similarity with ESWYT-5 and BARI GOM 28 at control. On the other hand the minimum vigour index were recorded from SATYN-20 (68.94, 16.64 at $0,5 \mathrm{dSm}^{-1}$, respectively). BARI GOM 29 scored 7.19 and 5.54 vigour index at 10 and $15 \mathrm{dSm}^{-1}$, respectively. At $20 \mathrm{dSm}^{-1}$ SATYN-22 and SATYN-25 wheat genotypes did not survive. Irrespective of vigour index, WICYT-7, SATYN-20 also showed sensitivity to salt stress condition. The higher vigour index attributed to higher germination rate and seedling length for salt tolerance cultivars. On the other hand the sensitive ones had the lower germination rate and seedling length, ultimately had a lower vigour index. Similar results were reported by Moud and Maghsoudi [30], Singh et al. [44] and Karim et al. [21].

\section{Conclusions}

Considering the above results obtaining from the present piece of work it may be concluded that among 33 wheat genotypes ESWYT-5, ESWYT-6 and BARI GOM 28 wheat genotypes were salt tolerance which were attributed to higher germination rate, shoot length, root length, shoot dry weight, root dry weight, relative water content, water retention capacity, coefficient of velocity and vigor index and rest of the wheat genotypes found to be moderately to strongly sensitive to salt stress.

\section{Acknowledgements}

This research work was fully financed by BAS-USDA, PALS, project no. CR-09, Bangladesh Academy of Science, National Science and Technology Museum Bhaban, Agargaon, Dhaka-1207, Bangladesh.

\section{REFERENCES}

[1] Abdul-Baki, A. A. and Anderson J. D. (1970). Viability and leaching of sugars from germinating barley. Crop Sci. 10: 31-34.

[2] Agnihotri, R. K., Palni, L. M. S. and Pandey, D. K. (2006). Screening of land races of rice under cultivation in Kumaun Himalayan for salinity stress during germination and early seedling growth. Indian J. Plant Physiol. 11(30): 262-272.
[3] Akbarimoghaddam, H., Galavi, M., Ghanbari, A. and Panjehkeh, N. (2011). Salinity effects on seed germination and seedling growth of bread wheat cultivars. Trakia J. Sci. 9(1): 43-50.

[4] Allakhverdiev, S. I., Sakamoto, A., Nishiyama, Y., Inaba, M. and Murata, N. (2000). Ionic and Osmotic effects of $\mathrm{NaCl}$-induced in activation of photo systems I and II in Synechoccus sp. Plant physiol. 123: 1047-56.

[5] Ashraf, M. and Harris, P. J. C. (2004). Potential biochemical indicators of salinity tolerance in plants. Plant Sci. 166: 3-16.

[6] Ashraf, M. and Khanum, A. (1997). Relationship between ion accumulation and growth in two spring wheat lines differing in salt tolerance at different growth stage. J. Agron. Crop Sci. 179: $39-51$.

[7] Ashraf, M. and Oleary, J. W. (1996). Responses of some newly developed salt-tolerant genotypes of spring wheat to salt stress. Yield components and ion distribution. J. Agron. Crop Sci. 176: 91-101.

[8] Ashraf, M., Foolad, M. R. (2005). Pre-sowing seed treatment-a shotgun approach to improve germination, plant growth and crop yield under saline and non-saline conditions. Adv. Agron. 88: 223-271.

[9] Baque, M. A., Karim, M. A. and Hamia, A. (2002). Role of potassium on water relation behavior of Triticum astivum L. under water stress conditions. Prog. Agric. 13(1\&2): 71-75.

[10] Bera, A. K., Pati, M. K. and Bera, A. (2006). Bassionolide ameliorates adverse effect on salt stress on germination and seedling growth of rice. Indian J. Plant Physiol. 11(2): 182-189.

[11] Bhatti, M. A., Ali, Z., Bakhsh, A., Razaq, A. and Jamali, A. R. (2004). Screening of wheat lines for salinity tolerance. Int. J. Agril. Bio. 6(4): 627-628.

[12] Cha-Um, S., Kirdmanee, C. and Supaibulwatana (2004). Biochemical and physiological responses of thai jasmine rice (Oryza sativa L. sp. Indica cv. KOML 105) to salt stress. Sci. Asia. 30: 247-253.

[13] Cherian, S. and Reddy, M. P. (2000). Salt tolerance in halophyte Scaedanudeflora. Effect of $\mathrm{NaCl}$ on growth, ion accumulation and oxidative enzymes. Indian J. Plant Physiol. 5(1): 32-37.

[14] Copeland, L. (1976). Principle of Seed Science and Technology. Burgess Publishing Company, Minneapolis, Mannishly. pp. 164-165.

[15] Cramer, G. R., Alberico, G. J. and Schmidt, C. (1994). Salt tolerance is not associated with the sodium accumulation of two maize hybrids. Australian J. Plant Physiol. 21: 675-692.

[16] Dagar, J. C., Bhagwan, H. and Kumar, Y. (2004). Effect of growth performance and biochemical content of Salvadora persica when irrigated with water of different salinity. Indian J. Plant Physiol. 9(3): 234-238.

[17] Datta, J. K., Nag, S., Banerjee, A. and Mondal, N. K. (2009). Impact of salt stress on five varieties of Wheat (Triticum aestivum L.) cultivars under laboratory condition. J. App. Sci. Environ. 13(3): 93-97.

[18] Flower, T. J., Salma, F. M. and Yeo, A. R. (1998). Water use efficiency in rice in relation to plant. Plant cell Environ. 11: 
453-459.

[19] Ghannadha, M. R., Omidi, M., Shahi, R. A. and Poustini, K. (2005). A study of salt tolerance in genotypes of bread wheat using tissue culture and germination test. Iranian J. Agri. Sci. 36 (1): $75-85$.

[20] Hasegawa, P. M., Breseen, R. A., Zhu, J. K. and Bohnert, H. J. (2000). Plant cellular and molecular responses to high salinity. Ann. Rev. Plant Physiol. Plant Molecular Bio. 51: 463-499.

[21] Karim, M. A., Utsunomiya, N. and Shigenaga, S. (1992). Effect of sodium chloride on germination and growth of hexaploid triticale at early seedling stage. Japan J. Crop Sci. 61(2): 279-284

[22] Khan, M. A., Ungar, I. A. and Showalter, A. M. (2000). Effects of sodium chloride treatments on growth and ion accumulation of the halophyte Haloxylon recurvum Commun. Soil Sci. Plant Anal. 31: 2763-2774.

[23] Khatkar, P. and Kuhad, M. S. (2000). Stage sensitivity of wheat cultivars to short terms salinity stress. Indian J. Plant Physiol. 5(1): 26-31.

[24] Kingsbury, R. W., Epstein, E. and Peorcy, R. W. (1984). Physiological responses to salinity in selected lines of wheat. Plant physiol. 74: 417-425.

[25] Kumar, A., Bandhu, P. and Das, A. (2005). Salt tolerance and salinity effects on plants: a review. Ecotoxicol. Environ. Saf. 60: 324-349.

[26] Lallu and Dixit, R. K. (2005). Salt tolerance of Mustard genotype at seedling stage. Indian J. Plant Physiol. 14(2): 33-35.

[27] Long, S. P. and Baker, N. R. (1986). Saline Terrestrial Environments. In: Baker, N.R., Long, S.P. (Eds.), Photosynthesis in Contrasting Environments, Elsevier, Amsterdam. pp. 63-102.

[28] Meiri, A. and Poljakoff-Mayber, A. (1970). Effect of various salinity regimes on growth, leaf expansions and transpiration rate of bean plants. Plant Soil Sci. 109: 26-34.

[29] Mirza, R. A. and Mahmood, K. (1986). Comparative effect of sodium chloride and sodium bicarbonate on germination, growth and ion accumulation in Phaseolus aureus, Roxb, c.v. 6601. Biologia. 32: 257-268.

[30] Moud, A. M. and Maghsoudi, K. (2008). Salt stress effects on respiration and growth of germinated seeds of different wheat (Triticum aestivum L.) cultivars. World J. Agril. Sci. 4(3): 351-358.

[31] Mujeeb, U. R., Soomro, U. A., Haq, M. Z. U. and Gul, S. (2008). Effects of $\mathrm{NaCl}$ salinity on wheat (Triticum aestivum L.) cultivars. World J. Agril. Sci. 4(3): 398-403.

[32] Munns, R. (2002).Comparative physiology of salt and water stress. Plant Cell Environ. 25: 239-250.
[33] Poustini, K. and Siosemardeh, A. (2004). Ion distribution in wheat cultivars in response to salinity stress. Field Crop Res. 85: $125-133$.

[34] Rahman, M., Kayani, S. A., Gul, S. (2000). Combined effects of temperature and salinity stress on corn cv. Sunahry. Pakistan J. Bio. Sci. 3(9): 1459-1463.

[35] Ramden, H. A., Niemi, S. A. and Hadathi, Y. K. A. (1986). Salinity and seed germination of corn and soy bean. Iraqui J. Agri. Sci. 4(2): 97-102.

[36] Rumena, Y. (2006). Physiology of rice genotypes under saltstress conditions. A PhD. Thesis. Department of Crop Botany, BSMRAU, Salna, Gazipur.

[37] Sairam, R. K., Veerabhadra, R. K. and Srivastava, G. C. (2002). Differential response of wheat genotypes to long term salinity stress in relation to oxidative stress, antioxidant activity and osmolyte concentration. Plant Sci. 163: 1037-1046.

[38] Sangakkara, U. R., Harteing, U. A. and Nosberger, J. (1996). Response of root branching and shoot water potentials of French bean (Phaseolus vulgaris L.) to soil moisture and fertilizer potassium. J. Agron. Crop Sci. 177: 165-173.

[39] Seeman, J. R. and Sharkey, J. D. (1986). Salinity and Nitrogen effects on photosynthesis, Ribulose-1, 5 bisphosphate carboxylase and metabolites pool size in Phaseolus vulgaris L. Plant physiol. 82: 555-560.

[40] Shannon, M. C. (1984). Breeding, selection and the genetics of salt tolerance. In: Staple, R.C., Toenniessen, G.H. (Eds.), Salinity Tolerance in Plants, 231-55, Wiley, New York.

[41] Sharma, P. C. (2003). Salt tolerance of Indian mustard: factor affecting growth and yield. Indian J. Plant Physiol. (Spl issue): 368-372.

[42] Shirazi, M.U., Asif, S. M., Khanzada, B., Khan, M. A., Ali, M., Mumtaj, S., Yousufazai, M. N. and Saif, M. S. (2001). Growth ion accumulation in some wheat genotypes under $\mathrm{NaCl}$ stress. Pakistan J. Biol. Sci. 4: 388-91.

[43] Sinclair, T. R. and Ludlow, M. M. (1985). Who thought plants thermodynamics? The unfulfilled potential of plant water potential. Australian J. Plant Physiol. 12: 213-217.

[44] Singh, A. K., Prakash, V. and Sastry, E. V. D. (2000). Effect of salinity stress on seed germination and seeding growth of wheat. Agric. Sci. Digestion. 20(2): 96-98.

[45] Soil Resources Development Institute (SRDI) (2010). Saline Soils of Bangladesh; SRDI, Ministry of Agriculture: Dhaka, Bangladesh.

[46] Tarmatt, A. and Munns, R. (1986).Use of concentrated micronutrient solution to separate osmotic from $\mathrm{NaCl}$ specific effects on plant growth. Australian J. Plant Physiol. 13: 509-522.

[47] Zhu, J. K. (2003). Regulation of ion homeostasis under salt stress. Curr. Opin. Plant Biol. 6: 441-445. 